\title{
A noninvasive swallowing measurement system using a combination of respiratory flow, swallowing sound, and laryngeal motion
}

\author{
Naomi Yagi ${ }^{1,2} \cdot$ Shinsuke Nagami ${ }^{1,2,3} \cdot$ Meng-kuan Lin $^{3} \cdot$ Toru Yabe $^{4}$. \\ Masataka Itoda ${ }^{5}$. Takahisa Imai ${ }^{6}$ Yoshitaka Oku ${ }^{3}$
}

Received: 12 November 2015 / Accepted: 2 September 2016 / Published online: 24 September 2016

(c) The Author(s) 2016. This article is published with open access at Springerlink.com

\begin{abstract}
The assessment of swallowing function is important for the prevention of aspiration pneumonia. We developed a new swallowing monitoring system that uses respiratory flow, swallowing sound, and laryngeal motion. We applied this device to 11 healthy volunteers and 10 patients with dysphagia. Videofluoroscopy (VF) was conducted simultaneously with swallowing monitoring using our device. We measured laryngeal rising time (LRT), the time required for the larynx to elevate to the highest position, and laryngeal activation duration (LAD), the duration between the onset of rapid laryngeal elevation and the time when the larynx returned to the lowest position. In addition, we evaluated
\end{abstract}

Naomi Yagi and Shinsuke Nagami have equally contributed to this work.

Yoshitaka Oku

yoku@hyo-med.ac.jp

1 Department of Neurology, Graduate School of Medicine, Kyoto University, 54 Shogoin-Kawaharacho, Sakyo-ku, Kyoto 606-8507, Japan

2 Clinical Research Center for Medical Equipment Development (CRCMeD), Kyoto University Hospital, 54 Shogoin-Kawaharacho, Sakyo-ku, Kyoto 606-8507, Japan

3 Department of Physiology, Division of Physiome, Hyogo College of Medicine, 1-1 Mukogawa-cho, Hyogo, Nishinomiya 663-8501, Japan

4 Murata Manufacturing Co., Ltd., 1-10-1, Higashikotari, Nagaokakyo, Kyoto 617-8555, Japan

5 Wakakusa Tatsuma Rehabilitation Hospital, 1580 Oaza-tatsuma, Daito, Osaka 574-0012, Japan

6 Ashiya Municipal Hospital, 39-1 Asahigaoka-cho, Ashiya, Hyogo 659-0012, Japan the coordination between swallowing and breathing. We found that LAD was correlated with a VF-derived parameter, pharyngeal response duration (PRD) in healthy subjects (LAD: $959 \pm 259 \mathrm{~ms}$ vs. PRD: $1062 \pm 149 \mathrm{~ms}$, $r=0.60$ ); however, this correlation was not found in the dysphagia patients. LRT was significantly prolonged in patients (healthy subjects: $320 \pm 175 \mathrm{~ms}$ vs. patients: $465 \pm 295 \mathrm{~ms}, P<0.001, t$ test). Furthermore, frequency of swallowing immediately after inspiration was significantly increased in patients. Therefore, the new device may facilitate the assessment of some aspects of swallowing dysfunction.

Keywords Swallowing - Dysphagia - Deglutition apnea . Coordination between swallowing and breathing

\section{Introduction}

Dysphagia, or swallowing difficulty, is the medical term for a condition in which the swallowing process is disrupted and eating ability is impaired. Patients with dysphagia can be at higher risk of pulmonary aspiration and subsequent aspiration pneumonia. According to WHO report in 2012, pneumonia was at third rank among causes of death in the world (World Health Organization-Fact sheets of Media Centre, The top 10 causes of death, http://www. who.int/mediacentre/factsheets/fs $310 /$ en/), and the majority of pneumonia cases in the elderly population are associated with aspiration. Swallowing abnormality may also contribute to exacerbations of pulmonary diseases $[4,10$, 13, 24, 33, 43, 44]. Recurrence of aspiration pneumonia frequently occurs if the underlying swallowing problems have not been properly treated. Therefore, the assessment of swallowing function and early intervention are critical 
for preventing the occurrence and recurrence of aspiration pneumonia.

There are several assessment methods that can be applied to evaluate patients with dysphagia. The two widely used bedside swallow assessment tests, repetitive saliva swallowing test (RSST) and modified water swallowing test (MWST), lack quantitative analyses. Currently, videofluoroscopy (VF) and videoendoscopy (VE) are considered the gold standard for evaluating dysphagia. However, VF cannot be conducted frequently since X-ray exposure may endanger the health of patients as well as medical staff. In addition, VF cannot be done outside the medical facility. VE is more portable than VF; however, specifically trained medical doctors (or dentists) must be available to diagnose the findings on site. Swallowing sound and motion analyses are alternative swallowing assessment techniques [1, 5, 22, 34]. However, in that motion analysis, 'motion' does not refer to that of the vocal cord, but rather it refers to the elevation of the larynx that causes the downward motion of the epiglottis to cover the airway to protect it during swallowing. Although they are safe, relatively simple, and easily repeatable, these techniques need to process acoustic or kinetic signals obtained by specially designed sensor devices, typically a laryngeal microphone or an accelerometer. Therefore, many researchers have developed algorithms to process these signals for the assessment of swallowing function $[8,22,27,36,42]$. To date however, there is still no sufficiently accurate and efficient way for objective monitoring of swallowing behavior in typical daily life environments. Therefore, we have devised a swallowing monitoring system that utilizes a combination of respiratory, acoustical and kinetic signals for more integrated monitoring and assessment of swallowing function. The rationale for the use of respiratory information is twofold: First, it serves as a good marker to detect swallows, since respiratory flow stops during swallowing (deglutition apnea). Secondly, it enables assessment of the coordination between swallowing and breathing, an important airway protection mechanism [28].

This paper mainly focuses on the method by which the new swallowing monitoring system detects swallowing events and assesses swallowing function from collected signal components, i.e., a respiratory flow, swallowing sound, and laryngeal motion. This paper extends the previous research work done by Yagi et al. [47].

In order to evaluate the efficiency and effectiveness of the newly developed monitoring system, we simultaneously monitored the VF measurement in both volunteer subjects and patients with dysphagia. We then compared the results obtained by our system and those by VF. We found that the newly developed method is able to accurately detect swallowing events and yield quantitative indices that may facilitate the assessment of some aspects of swallowing dysfunction.

\section{Materials and methods}

\subsection{Recorded components}

Three signal components were recorded by the swallowing monitoring system to detect and evaluate swallowing activity. Respiratory flow was measured by a nasal cannula-type flow sensor (Pro-Tech ProFlow cannula, Sleep Lab Products, USA) and a differential pressure transmitter (KL-17, Nagano Keiki, Japan) and recorded at $1 \mathrm{kHz}$. Laryngeal motion and swallowing sound were simultaneously recorded using a custom-made, piezoelectric sensor attached on the thyroid cartilage (Fig. 1b). The sensor is a piezoelectric film (the size of the detector is $10 \mathrm{~mm} \times 30 \mathrm{~mm}$ ), which generates electric charge upon bending. The sensor has a wide dynamic range between $0 \mathrm{~Hz}$ and $4 \mathrm{kHz}$. This sensor was custom made, as to our knowledge there was no commercially available film sensor with a sufficient dynamic range. The signal was amplified and divided into high $(>100 \mathrm{~Hz})-$ and low $(<100 \mathrm{~Hz})$-frequency components using high-pass and low-pass preamplifiers, respectively. This cutoff frequency was set to eliminate low-frequency interferences such as heart sounds and muscle artifacts from the (high frequency) sound component [35]. The $100 \mathrm{~Hz}$ cutoff frequency also assures the delay characteristics of the lowfrequency motion signal component within $30 \mathrm{~ms}$ for the frequency range of $0.5-10 \mathrm{~Hz}$, which minimizes the error in estimating the timing of laryngeal elevation in relation to the respiratory phase. Thus, the high-frequency component represents sound signal greater than $100 \mathrm{~Hz}$, whereas the lowfrequency component contains both laryngeal motion signal and low-frequency $(20-100 \mathrm{~Hz})$ sound signal. However, the
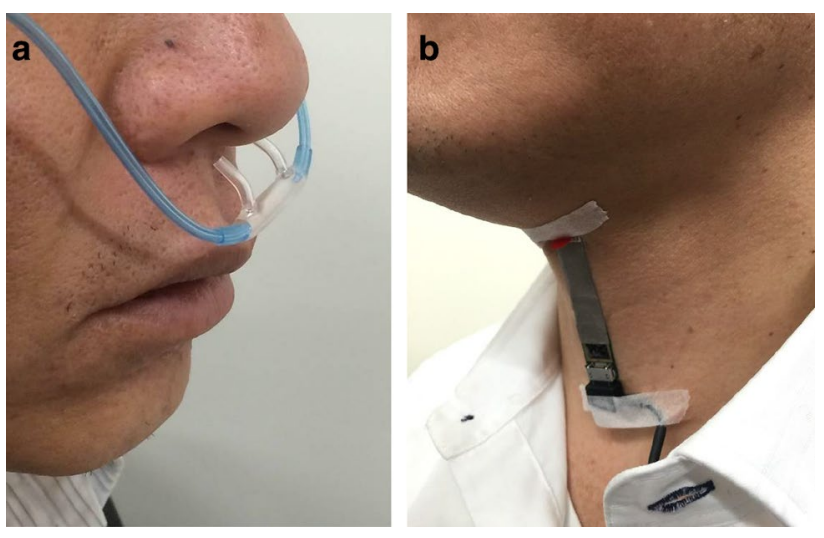

Fig. 1 Sensor devices. a A nasal cannula-type flow sensor is positioned near the nostril. b A piezoelectric sensor is affixed to the surface of the thyroid cartilage using an adhesive tape 
power spectrum density of this low-frequency component during swallow typically has a peak at $\sim 1 \mathrm{~Hz}$ and decreases toward the audible $(>20 \mathrm{~Hz})$ frequency range. Therefore, we assume that the low-frequency component mostly represents the laryngeal motion (as well as infrasound vibration) signal and refer to it as 'the laryngeal motion signal' hereafter. The laryngeal motion was recorded at $1 \mathrm{kHz}$, and the sound signal was recorded at $10 \mathrm{kHz}$ and stored simultaneously with the respiratory signal in a Micro SD card for later analyses. In addition, we recorded the timings of a swallow for later verification using a foot switch to generate TTLlevel pulse signals. The signals were analyzed using MATLAB (R2014b, Mathworks, USA) on a 64-bit Windows 8 professional-based computer.

\subsection{Experimental protocol}

Eleven healthy volunteers ( 9 males and 2 females, $40.1 \pm 10.7$ years old) and ten patients with dysphagia (4 males and 6 females, $75.6 \pm 9.4$ years old) were enrolled in this study. The severity of dysphagia in these patients was assessed using the Food Intake LEVEL Scale (FILS) [12]. Eight patients were at level 3 (swallowing training using a small quantity of food is performed), and two patients were at level 7 (easy-to-swallow food is orally ingested in three meals. No alternative nutrition is given). In seven healthy subjects and in ten patients, swallowing and respiration during videofluoroscopic measurements were simultaneously recorded using two sensor devices (Fig. 1). A nasal cannula-type flow sensor was attached to the nostril (Fig. 1a), and a piezoelectric sensor was placed on the skin surface around the thyroid cartilage with an adhesive tape (Fig. 1b). Each subject was instructed to swallow three types of test food (level 0 , level 2, and level 3 ) and water, two times, during simultaneous swallowing monitoring and VF. A nonionic contrast agent, iopamidol (Oypalomin-370, Konica Minolta, Japan), was mixed into these test foods so that it was diluted twofold (iodine concentration: $185 \mathrm{mg} / \mathrm{mL}$ ). The physical properties, e.g., viscosity, adhesiveness, and cohesiveness, of the test foods were precisely controlled (Table 1 ). The composites of level 0 , level 2, and level 3 were similar to soft jelly, hard jelly, and paste, respectively. We did not add the contrast agent to the water. This protocol was approved by local ethical committees of Hyogo College of Medicine (No. 1580 and No. 1636), Kyoto University (No. C819 and No. C820), Takahashi Hospital, and Wakakusa Tatsuma Rehabilitation Hospital.

\subsection{Respiratory flow component processing}

In order to improve the detection accuracy and enhance the functional evaluation of swallowing activity, we
Table 1 Test food texture

\begin{tabular}{lcll}
\hline & $\begin{array}{l}\text { Hardness } \\
\left(\mathrm{N} / \mathrm{m}^{2}\right)\end{array}$ & $\begin{array}{l}\text { Cohesiveness } \\
\left(\mathrm{J} / \mathrm{m}^{3}\right)\end{array}$ & Adhesiveness \\
\hline $\begin{array}{l}\text { Coffee taste } \\
\text { Level 0 }\end{array}$ & $5104 \pm 354$ & $14 \pm 11$ & $0.262 \pm 0.015$ \\
Level 2 & $11,618 \pm 846$ & $10 \pm 1$ & $0.430 \pm 0.060$ \\
Level 3 & $451 \pm 16$ & $83 \pm 9$ & $0.862 \pm 0.015$ \\
Orange taste & & & \\
Level 0 & $4682 \pm 247$ & $40 \pm 11$ & $0.246 \pm 0.021$ \\
Level 2 & $11,414 \pm 596$ & $24 \pm 6$ & $0.292 \pm 0.017$ \\
Level 3 & $476 \pm 19$ & $74 \pm 7$ & $0.808 \pm 0.012$ \\
\hline
\end{tabular}

* Measurement temperature: $20 \pm 2{ }^{\circ} \mathrm{C}$

analyzed respiratory activity. According to our previous study [47], an analysis using the combination of swallowing sound and breathing information could increase the accuracy of extracting swallows. Furthermore, the analysis of respiratory activity before and after swallowing is particularly useful and important for patients who have limited ventilatory capacity because breathing is closely coordinated with swallowing activity [17, 20, 40]. First, we classified respiratory activity into three phases: inspiration, expiration, and pause. A pause where no respiratory flow signal is detected may be considered as a deglutition apnea, but it might also be a voluntary cessation of breathing; thus, the two must be differentiated based on the presence of characteristic sound and motion (see Sect. 2.5). The deglutition apnea is an important airway defensive reflex to avoid an aspiration, where breathing is temporarily stopped during deglutition. This respiratory cessation period can be considered as a marker to identify swallowing activity. The algorithm for analyzing respiratory phases first searches for inspiratory-to-expiratory (I-E) transition and expiratory-to-inspiratory (E-I) transition. Then, a pause within a breath is detected as a period where the respiratory flow signal falls within a certain range around the zero-flow level. If the pause duration is greater than $0.35 \mathrm{~s}$, it is considered as a candidate of deglutition apnea. Figure 2 shows examples of respiratory phase discrimination before and after swallowing events using developed algorithms. As shown in Fig. 2a, a brief inspiratory flow signal is often recorded immediately after swallowing. However, this is not a true inspiration, but rather it is a negative pressure associated with the relaxation of the pharyngeal constrictor muscle; it is called swallow non-inspiratory flow (SNIF) [3]. To discriminate between a true inspiration and SNIF, we have set a minimal inspiratory time for a negative pressure swing to be considered as an inspiration, and the program searches the next inspiratory activity if the inspiratory time is less than $0.3 \mathrm{~s}$. These parameter values were set empirically. 


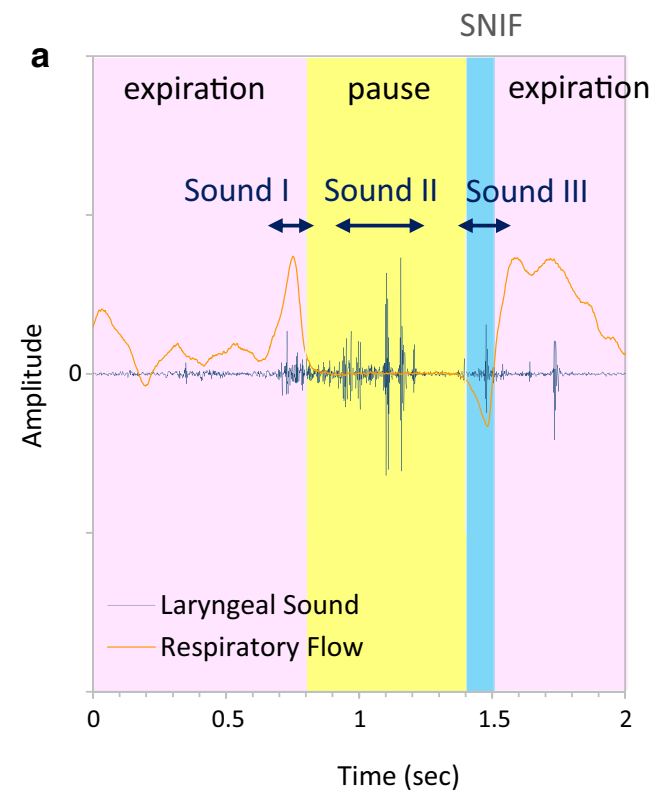

Fig. 2 Representative swallow-respiratory coordination patterns are presented together with swallowing sounds (Sounds I, II, and III). Light pink zone indicates the expiratory phase, light yellow zone, the pause phase, and light green zone, the inspiratory phase. a Expira-

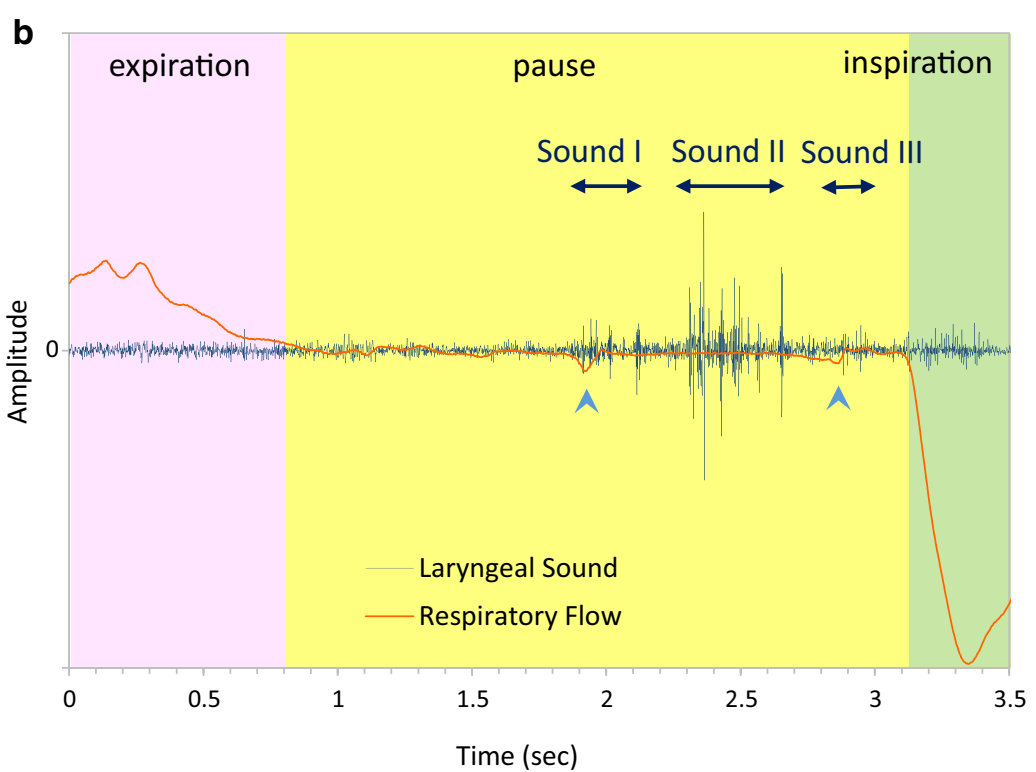

tion-swallow-expiration pattern. Blue zone represents swallow noninspiratory flow (SNIF). b Expiration-swallow-inspiration pattern. Note that small negative pressures (arrowheads) are recorded coincident with swallowing sound components
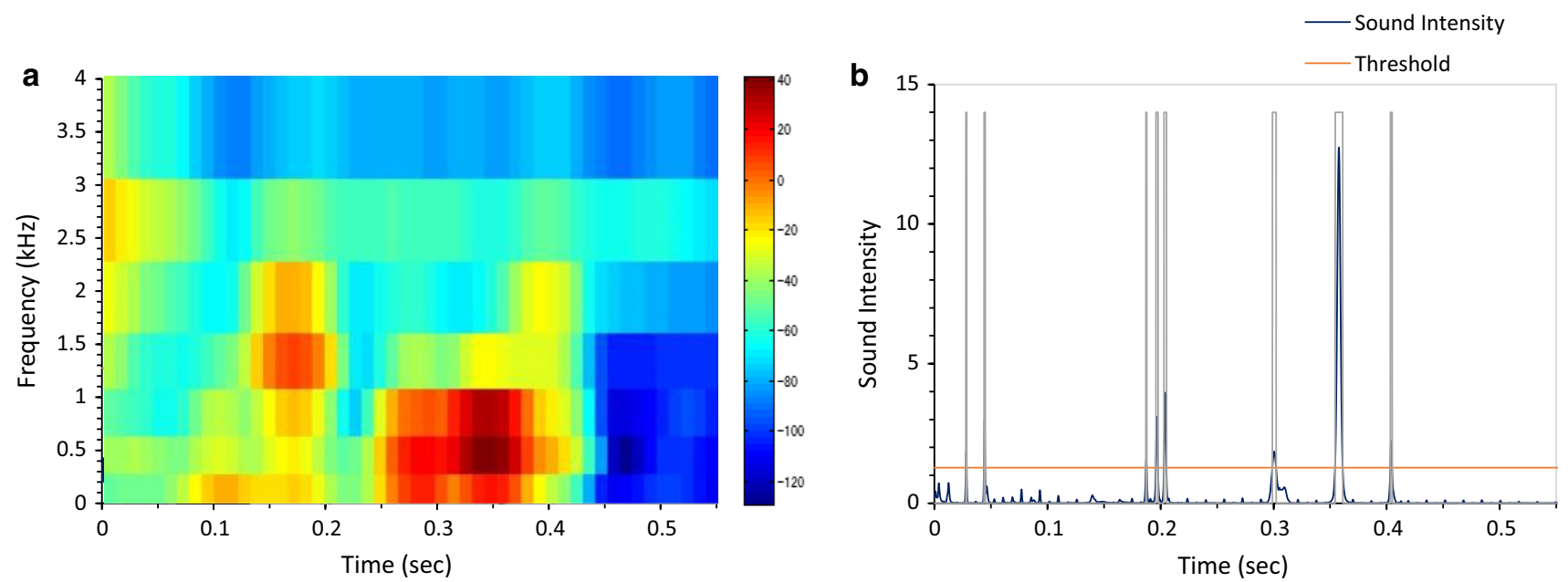

Fig. 3 Laryngeal sound is decomposed into a the time-frequency domain and $\mathbf{b}$ sound pulses

\subsection{Sound component processing}

Frequency analyses applied to the sound for swallowing detection are shown in Fig. 3. The program first calculates periodogram to estimate the power spectral density. While a simple waveform is a one-dimensional representation of sound, the two-dimensional representation describes the acoustic signal as a time-frequency image. We next applied mel-frequency cepstral coefficients (MFCCs), which is a representation of the short-term power spectrum
[6] to those images. This way, we can extract features of swallowing sound characteristics effectively [39]. This technique combines an auditory filter bank with a cosine transform, which provides a rate representation roughly similar to the auditory system. Swallowing sound typically contains a high-frequency component greater than $750 \mathrm{~Hz}$ [39], whereas vesicular and bronchial sounds consist of lower $(<500 \mathrm{~Hz})$-frequency sounds [26]. Therefore, the program searches for particular sound data that generated specific high-frequency bands during monitoring. Several 
frequency decomposition methods have been used for analysis of non-stationary signals such as continuous wavelet transform (CWT) and short-time Fourier transformation (STFT). We used STFT with an epoch duration of $1.5 \mathrm{~s}$ and a step size of $0.2 \mathrm{~s}$ for presenting the whole data, STFT with an epoch duration of $0.1 \mathrm{~s}$ and a step size of $0.01 \mathrm{~s}$ for presenting the sound signal within respiratory cessation periods (Fig. 3a), and CWT for feature extraction. The CWT was computed using the Morlet wavelet.

The sound data during respiratory cessation periods are retrieved, and the percent power of $500-2300 \mathrm{~Hz}$ frequency bands is calculated for each sound signal during epochs. If the percent power of $500-2300 \mathrm{~Hz}$ frequency bands is less than $20 \%$, then we determined that it is less likely to be a swallow according to the sensor characteristics. The sound signal was then decomposed into pulses to obtain two parameters, the number of pulses and the maximal pulse width (Fig. 3b). We defined and discriminated swallowing sound characteristics from those parameters. If the number of pulses is greater than 20 , or if the maximal pulse width is greater than $40 \mathrm{~ms}$, then it is considered to be an artifact or noise.

A normal swallowing sound typically consists of three sound components [46]. The first sound (Sound I) and the third sound (Sound III) are not always detected; however, the second component (Sound II) is consistently and most remarkably audible among three swallowing sound components [25]. Therefore, the program searches the time point of the most prominent sound power peak within each respiratory cessation period to identify the possible Sound II (Fig. 2).

\subsection{Detection of swallowing candidate periods}

In order to extract swallowing activity, we use deglutition apnea, swallowing sound characteristics, and amplitude of laryngeal motion. We propose a knowledge-based approach to discriminate between swallowing sounds and noises. The swallowing candidate period detection algorithm is shown in Fig. 4.
Fig. 4 Flow chart of the swallowing detection algorithm

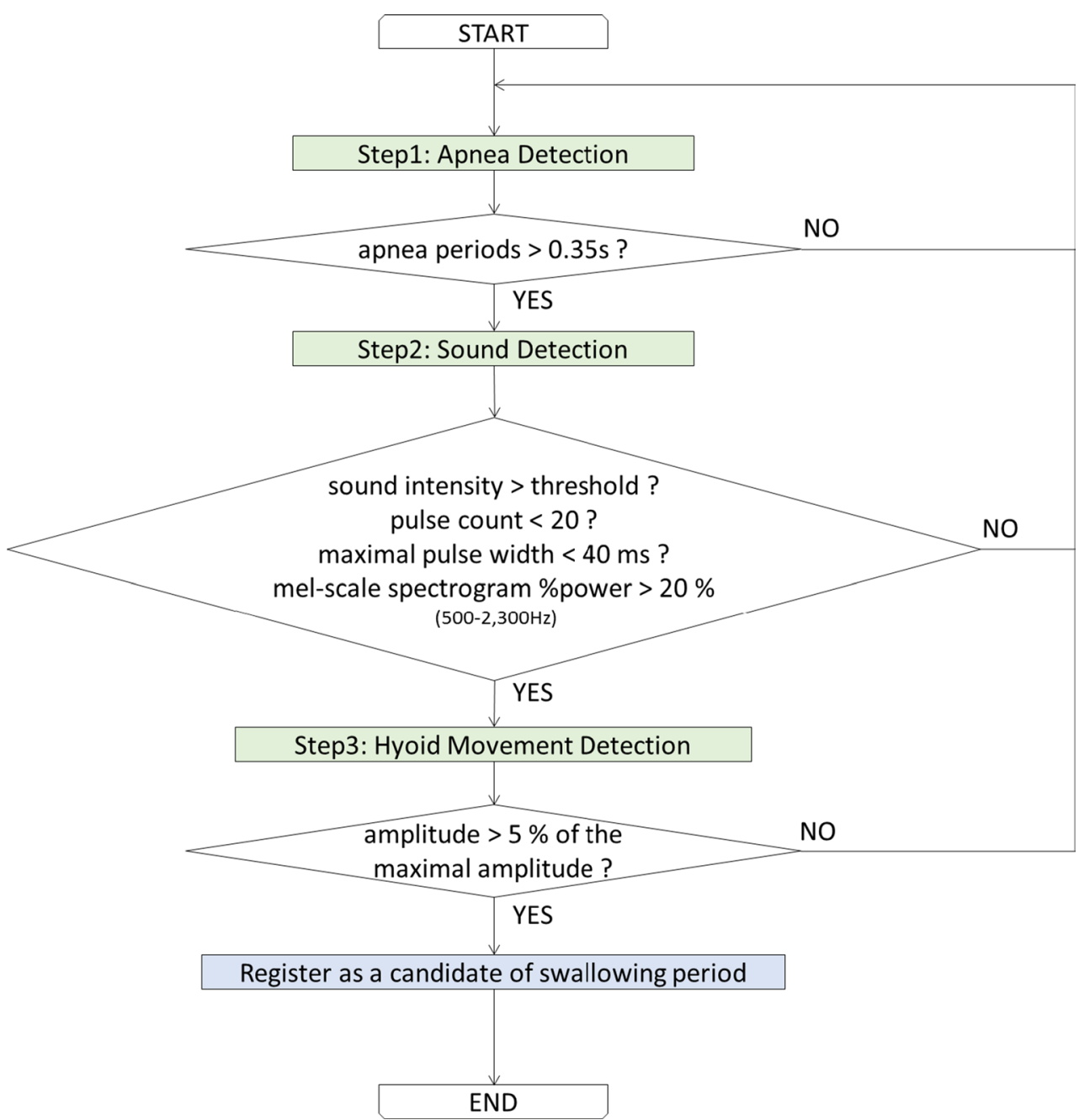


a

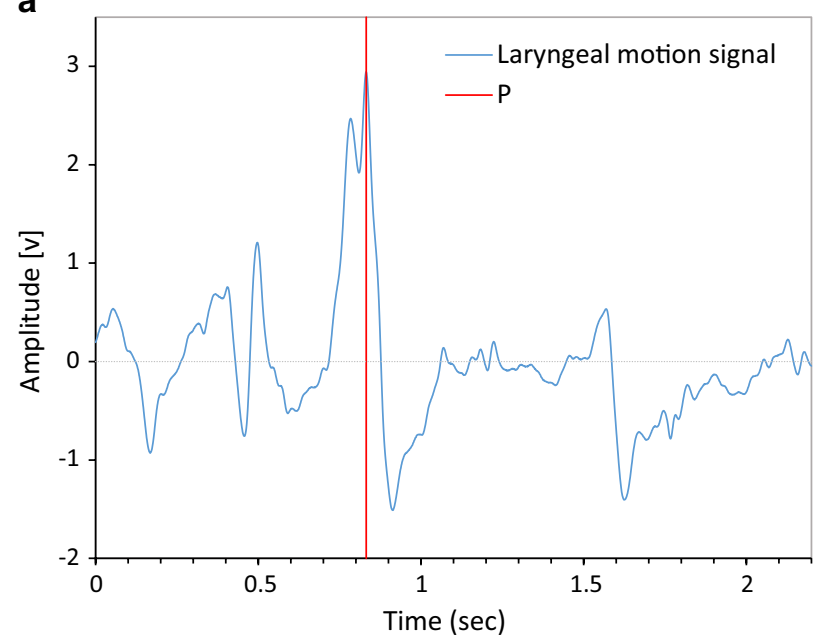

Fig. 5 a Raw sensor output. The program detects the time point $P$ (red line) at which the sensor output reaches the peak during the laryngeal elevation and the zero-cross point is searched backward and forward to identify the start point (the trough $T_{1}$ in $\mathbf{b}$ ) and end point (the maximum $M$ in b) of laryngeal rising time (LRT). b Integrated sensor signal (gray). The green line $P$ corresponds to the position of

First respiratory cessation periods $(>0.35 \mathrm{~s})$ are extracted (Step 1). If an extracted period contains sound whose intensity is greater than a certain threshold (e.g., noise level $+2 \times$ standard deviation), proceed to further steps (Step 2). In the next step, sound characteristics are analyzed as described above. If the pulse count is $<20$ counts, the maximal pulse width of which is $<40 \mathrm{~ms}$, and melscale spectrogram \%power within $500-2300 \mathrm{~Hz}$ frequency bands is $>20 \%$, and it is associated with laryngeal motion of amplitude $>5 \%$ of the maximal amplitude within the entire record; then, the extracted period is registered as a candidate of swallowing period.

\subsection{Laryngeal motion component processing}

Evaluating laryngeal motion is critical for assessing the swallowing function. During swallowing, the hyoid bone traces a path upward and forward and then returns to the original position, and the laryngeal prominence (the thyroid cartilage) traces a trajectory closely linked to that of the hyoid bone [41]. Therefore, the motion data are extracted based on the displacement of the laryngeal prominence. Here, we provide two temporal parameters to evaluate the swallowing function. Since the piezoelectric sensor has a differential characteristic against bending, it produces a positive signal associated with the laryngeal elevation and a negative signal associated with the laryngeal descent

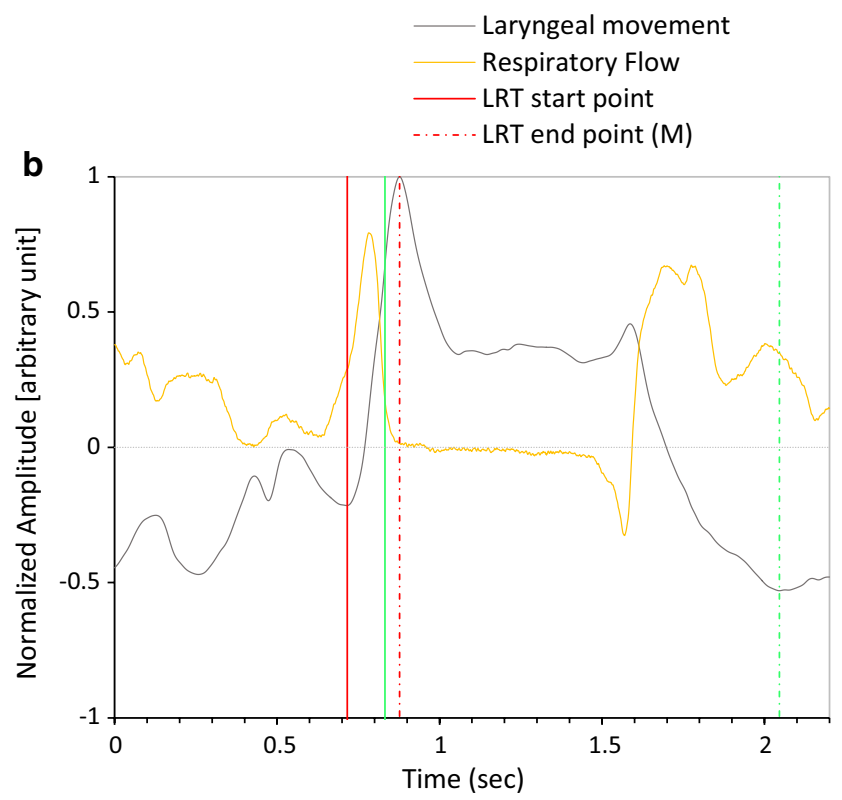

the highest peak in $\mathbf{a}$, in which the laryngeal elevation speed becomes maximal. The duration between the trough $T_{1}$ (solid red line) and the peak $M$ (dashed red line) is the duration of LRT. The duration between the time point $P$ and the trough $T_{2}$ is the duration of laryngeal activation duration (LAD)

(Fig. 5a). Therefore, we first integrated the piezoelectric signal to estimate the laryngeal motion (Fig. 5b):

$\mathrm{ILM}=\int \mathrm{LM}(t)-\overline{\mathrm{LM}}$

where LM is the raw laryngeal motion signal and ILM is the integrated laryngeal motion signal. However, the integrated piezoelectric signal does not completely match the motion of the thyroid cartilage. Therefore, we define two parameters that characterize the shape of the integrated piezoelectric signal and compare them with parameters that characterize the dynamics of swallowing on videofluoroscopy.

\subsection{Laryngeal rising time (LRT)}

Slow laryngeal elevation may cause a delay of laryngeal closure for airway protection during the pharyngeal phase of swallowing. Therefore, we define laryngeal rising time (LRT) as the time required for the larynx to elevate to the highest position (Figs. 5, 6).

Within each identified respiratory cessation period, the program first searches for the time point $(P)$ at which the sensor output (LM) reaches the highest peak. Due to the differential characteristics of the piezoelectric sensor, this corresponds to the instance when the laryngeal elevation speed becomes maximal. Since the time point 

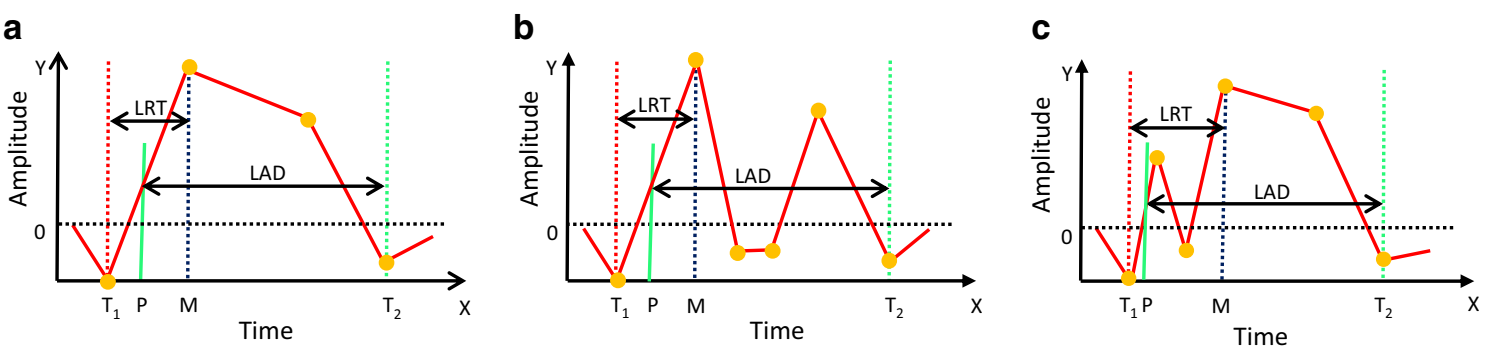

Fig. 6 Representative ILM trajectory patterns during swallowing. a Standard monotonous laryngeal elevation activity, b laryngeal activity drops and passes the zero-cross level after reaching the maximal elevation, and c laryngeal activity drops and passes the zero-cross level before reaching the maximal elevation. The duration between

$P$ corresponds to the onset of pharyngeal swallow, which usually occurs after the onset of respiratory cessation [20], and Sound II is associated with bolus transit [46], we limited the possible position of $P$ to be the range between (the onset of deglutition apnea-200 ms) and (Sound II $+200 \mathrm{~ms}$ ). Then, we defined $P$ as the local maxima within this range (Fig. 5a).

Next, the program searches the zero-cross point backward to estimate the start point of LRT $\left(T_{1}\right)$. If ILM at this zero-cross point has a positive value, then the backward search is continued until the local minima of ILM with a negative value are found. The program then looks for the time point $M$ where the larynx reaches the maximal elevation (Fig. 5b). $M$ corresponds to the first zero-cross point in LM searched forward from $P$. Finally, LRT is calculated as the duration between $T_{1}$ and $M$. Due to the varying structure of swallowing pattern recorded from subjects with different swallowing functions and different postures, several types of ILM patterns can be observed (Fig. 6). Therefore, if LRT is less than $45 \mathrm{~ms}$, then the program finds the second highest LM peak within the range between (the onset of deglutition apnea-200 ms ) and (Sound II $+200 \mathrm{~ms}$ ) and repeats the LRT calculation until LRT $>45 \mathrm{~ms}$.

\subsection{Laryngeal activation duration (LAD)}

We next define laryngeal activation duration (LAD) as the duration between the time point $P$ and the time point at which the integrated sensor output becomes the trough $\left(T_{2}\right)$ during the descent of the larynx (Figs. 5 and 6). Since LAD represents the duration of the pharyngeal swallow, LAD should be greater than $500 \mathrm{~ms}$; otherwise, the next trough is searched forward.

We set minimal values for LRT and LAD, since ILM sometimes displayed zero-cross activity patterns (Fig. 6b, c). Zero-cross activity patterns were often observed associated with extension and flexion of the head of subjects, the trough $T_{1}$ (red dotted line) and the peak $M$ (blue dotted line) is the duration of laryngeal rising time (LRT). The duration between the time point $P$ (at which the laryngeal elevation speed becomes maximal) and the trough $T_{2}$ is the duration of laryngeal activation duration (LAD)

since such swallowing maneuvers cause the piezoelectric sensor to bend and generate a signal which overlaps with the laryngeal motion signal.

\subsection{Swallowing simulator}

In order to assess the characteristics of the newly developed piezoelectric sensor, we created a device that simulates the motion of the thyroid cartilage during swallowing (Fig. 7ac). The device, hereafter termed 'swallowing simulator,' has a motorized pushing mechanism, whose two-dimensional position is controlled by two linear actuators (servomotors and precise screws) that, respectively, simulate forwardbackward and upward-downward motion of the thyroid cartilage. Since the major frequency band of laryngeal motion is approximately $1 \mathrm{~Hz}$, we tested responses of the piezoelectric sensor to $1 \mathrm{~Hz}$ sinusoidal movements in the forward-backward (Fig. 7d) and upward-downward (Fig. 7e) directions and with a combination of the two (Fig. 7f); we verified that the sensor responded linearly to $1 \mathrm{~Hz}$ sinusoidal movements of both the forward-backward and upward-downward directions and also the combination of the two directions, with 40-80ms delay.

\subsection{Videofluorographic (VF) measurements}

Swallowing function is often assessed by temporal parameters measured using videofluoroscopic images. Among these parameters, we measure the pharyngeal response duration (PRD) [15] and laryngeal elevation delay time (LEDT) [23]. PRD reflects dynamics of the hyoid bone during swallowing. The hyoid bone slowly elevates posteriorly before the initiation of the swallowing reflex and then rapidly starts moving forward upon initiation of the swallowing reflex (pharyngeal swallow) to elevate the larynx. When suprahyoid muscles relax and infrahyoid muscles are activated, the hyoid bone moves backward and downward 
a

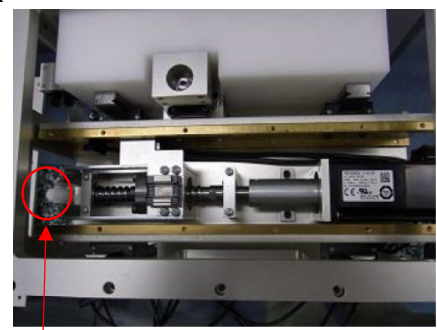

pusher b

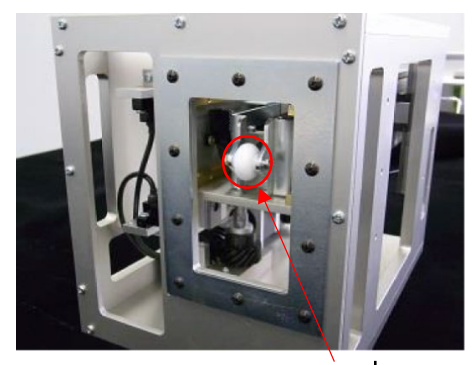

pusher

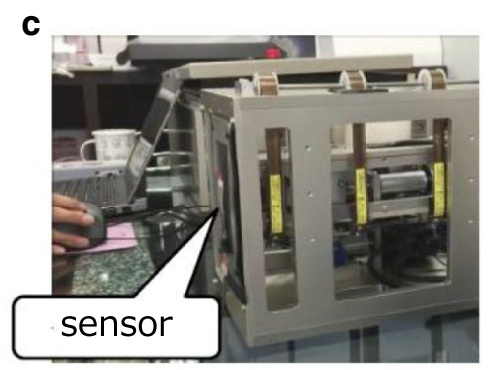

f

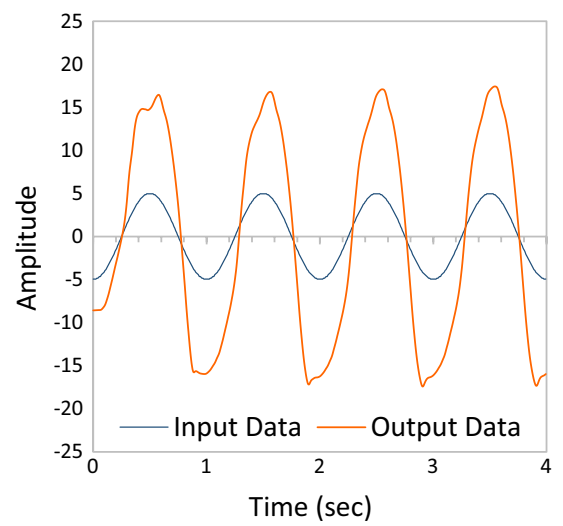
sensor d

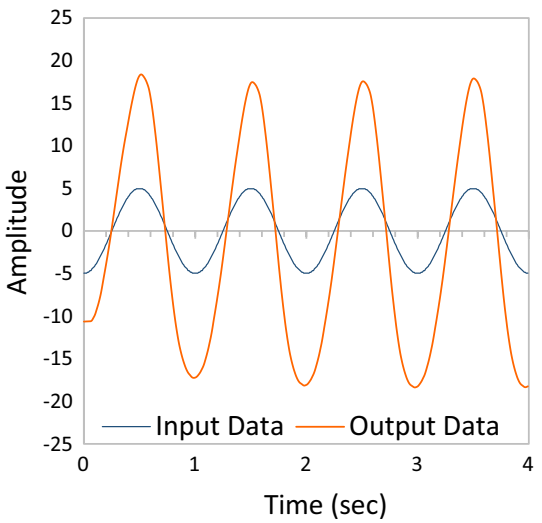

e

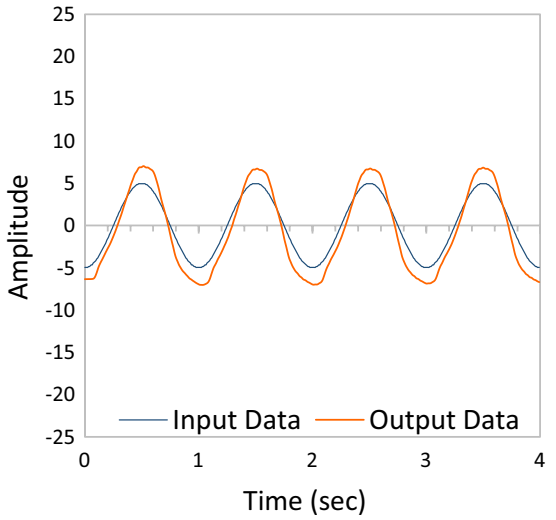

Fig. 7 Swallowing simulator that simulates the forward-backward and the anterior-posterior motion of the thyroid cartilage by two linear actuators. a Simulator top view, b simulator side view, c sensor was placed on the pusher, $\mathbf{d}$ forward-backward input data, $\mathbf{e}$ anteriorposterior input data, $\mathbf{f}$ combination input data

To compensate for movement of the body, the $\mathrm{X}-\mathrm{Y}$ coordinates of the anterior-inferior edge of the $\mathrm{C} 4$ vertebral body were also measured, which served as the anchor point. Then, the anterior and vertical displacements of the hyoid bone were calculated according to the method described by Kim and McCullough [11].

\subsection{Statistics}

Occurrence rates of specific coordination patterns between swallowing and breathing in healthy subjects and in patients with dysphagia were compared using Chi-square test. The swallowing characteristics of different food textures/levels were tested using ANOVA. Correlations between the parameters derived from the new device and VF-derived parameters were evaluated by Pearson's correlation coefficient. LRT and LAD values in healthy subjects and patients were compared using unpaired $t$ test, with all data presented as mean \pm standard deviation. $P$ values were two-sided, and $P<0.05$ was considered as statistically significant. Statistical analyses were performed using JMP Pro, SAS Institute Inc. (version 12). 


\section{Results}

\subsection{Accuracy of semiautomatic swallowing detection}

The accuracy of semiautomatic swallowing detection was assessed using data from 7 healthy subjects, for whom two speech therapists judged swallow candidates. First, swallowing candidate periods were automatically extracted using the algorithm described in Methods. The automatic detection algorithm picked up 94 swallow candidate periods from 7 subjects, which included 55 test food swallowing periods (confirmed by the timing coincident with foot switch signals) and 39 additional dry (saliva) swallowing candidate periods. Since each subject swallowed test foods eight times, the sensitivity of the automatic swallowing detection algorithm with regard to test food swallows was $55 /(7 \times 8)=0.982$.

At this point, additional swallowing candidate periods contain false-positive detections (non-swallowing sounds) due to environmental noises, e.g., speech, motion artifacts, and electrical interference. Subsequently, the sound within these respiratory cessation periods (swallowing candidate periods) was played back, and two speech therapists independently judged whether the sound and the laryngeal motion (Fig. 5b) were compatible with a swallow. Each speech therapist judged 28 candidates as dry swallows (true positives) and 11 candidates as non-swallowing sounds (false positives). The judgment perfectly matched, and thus, the Cohen's kappa coefficient was 1.0. Therefore, the specificity of the automatic swallowing detection algorithm was $(94-11) / 94=0.883$. When we use only the laryngeal motion characteristics to detect swallows, the sensitivity with regard to test food swallows was 1.0, and the specificity was 0.712 .

\subsection{Characteristics of swallows}

Normal swallows in healthy subjects were accompanied by deglutition apneas, the duration of which was $1441 \pm 1152 \mathrm{~ms}$ (range $302-5834 \mathrm{~ms}$ ). It is known that, in general, typical swallows occur during expiration and are followed by expiration (E-SW-E pattern; Fig. 2a). However, in healthy subjects, 4 of 98 swallows occurred during inspiration (I-SW pattern), and 5 of 98 swallows were followed by inspiration (SW-I pattern; exemplified in Fig. 2b). In patients with dysphagia, the duration of deglutition apneas was $2386 \pm 2089 \mathrm{~ms}$ (range 375-11,599 ms), 7 of 46 swallows occurred during inspiration, and 6 of 46 swallows were followed by inspiration. The occurrence rate of I-SW pattern but not SW-I pattern was significantly increased in the patient group (Chi-squared test of proportion, I-SW: $P=0.019$ and SW-I: $P=0.094)$.
The respiratory cycle is reset by a swallowing event, and the timing of initiation of a new inspiration depends on the timing when within the respiratory cycle, the swallowing event occurs [32]. Therefore, we evaluated the phase-response relationship. According to the definition of Paydarfar et al. [32], we calculated the 'old phase' as the timing of swallowing from the onset of preceding inspiration and the 'co-phase' as the interval between the onset of swallowing and the onset of the subsequent inspiration. Both the old phase and co-phase are normalized by the mean respiratory cycle duration. Figure 8 shows the phaseresponse curve (co-phase plot), in which the respiratory rhythm was reset by swallowing events. The co-phase was large and variable for swallows initiated near the I-E transition. It should be noted that some swallows occurred after a complete respiratory cycle elapsed (old phase $>1$ ); this is thought to be due to the delay in onset of these swallows caused by the chew-swallow complex behavior. These phase-response characteristics did not differ between the healthy subjects and patients.

Normal swallowing sounds in healthy subjects consisted of $6 \pm 4$ pulses (range 1-20 pulses), the maximal pulse width of which was $8.1 \pm 5.8 \mathrm{~ms}$ (range $2.4-33.3 \mathrm{~ms}$ ), and mel-scale spectrogram \%power within $500-2300 \mathrm{~Hz}$ frequency bands was $71.1 \pm 21.9 \%$ (range $21.5-97.3 \%$ ). In patients with dysphagia, swallowing sounds consist of $7 \pm 4$ pulses (range 1-15 pulses), the maximal pulse width of which was $9.1 \pm 7.4 \mathrm{~ms}$ (range 2.4-34.4 ms), and melscale spectrogram \%power within $500-2300 \mathrm{~Hz}$ frequency bands was $57.3 \pm 16.0 \%$ (range 26.4-93.4\%).

\subsection{Estimation of swallowing function}

Following the semiautomatic swallowing detection, we estimated LRT and LAD and compared those with LEDT and PRD derived from videofluoroscopic image analysis. Since water did not contain a contrast agent, LEDT was not measured for water swallows.

In healthy subjects, LRT and LAD were $320 \pm 175 \mathrm{~ms}$ (range 99-1136 ms) and $959 \pm 259 \mathrm{~ms}$ (range 579$1699 \mathrm{~ms}$ ), respectively. On the other hand, LEDT and PRD were $201 \pm 56 \mathrm{~ms}$ (range 132-297 ms) and $1062 \pm 149 \mathrm{~ms}$ (range 835-1505 ms), respectively. In patients with dysphagia, LRT and LAD were $465 \pm 295 \mathrm{~ms}$ (range 151-1583 ms) and $886 \pm 311 \mathrm{~ms}$ (range 507-1799 ms), respectively. On the other hand, LEDT and PRD were $273 \pm 124 \mathrm{~ms}$ (range 99-570 ms) and $1292 \pm 243 \mathrm{~ms}$ (range $870-1870 \mathrm{~ms}$ ), respectively. The swallowing characteristics of different food textures/levels did not differ in healthy subjects and patients. The correlation coefficient between LRT and LEDT was 0.10 in healthy subjects and 0.07 in patients and that between LAD and PRD was 0.6 in 


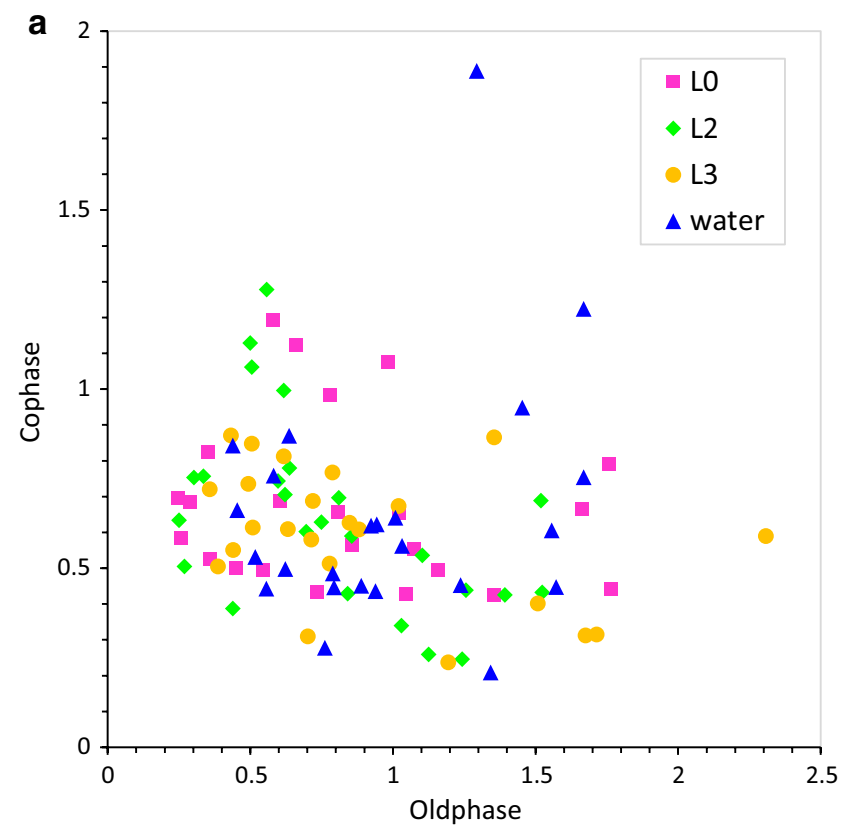

Fig. 8 Phase-response curve (co-phase plot) showing how the respiratory rhythm is reset by swallowing events depending on the timing of swallowing. I-SW represents a case where a swallow occurred

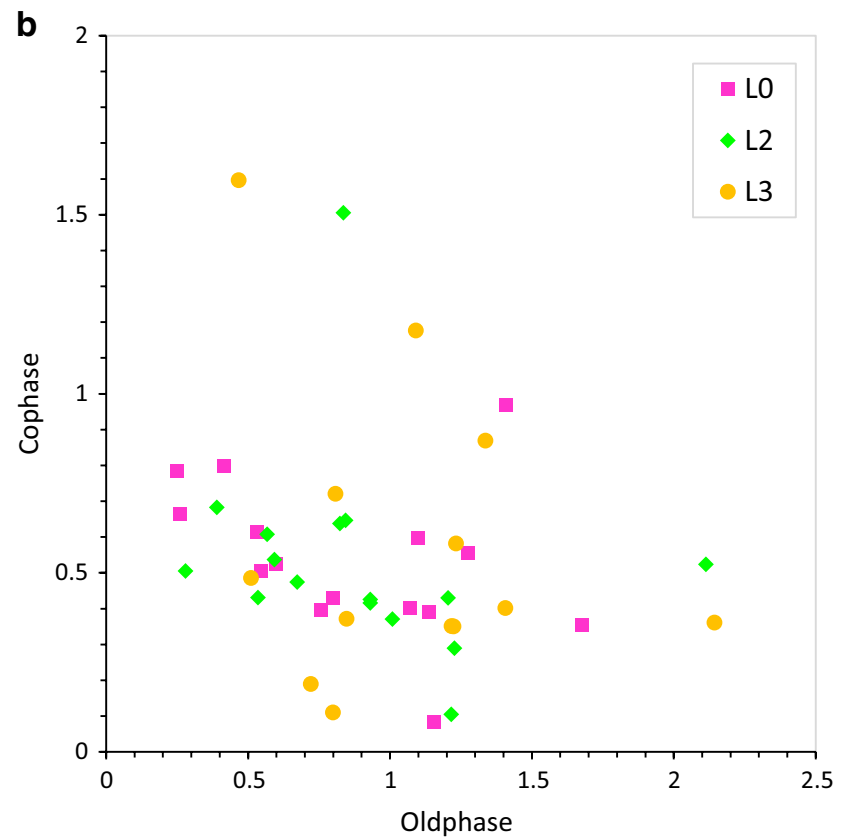

during inspiration, and SW-I represents a case where a swallow was followed by inspiration. a Healthy subjects, b patients. One outlier (old phase $=5.85$, co-phase $=0.70, \mathrm{~L} 0$ ) is not plotted
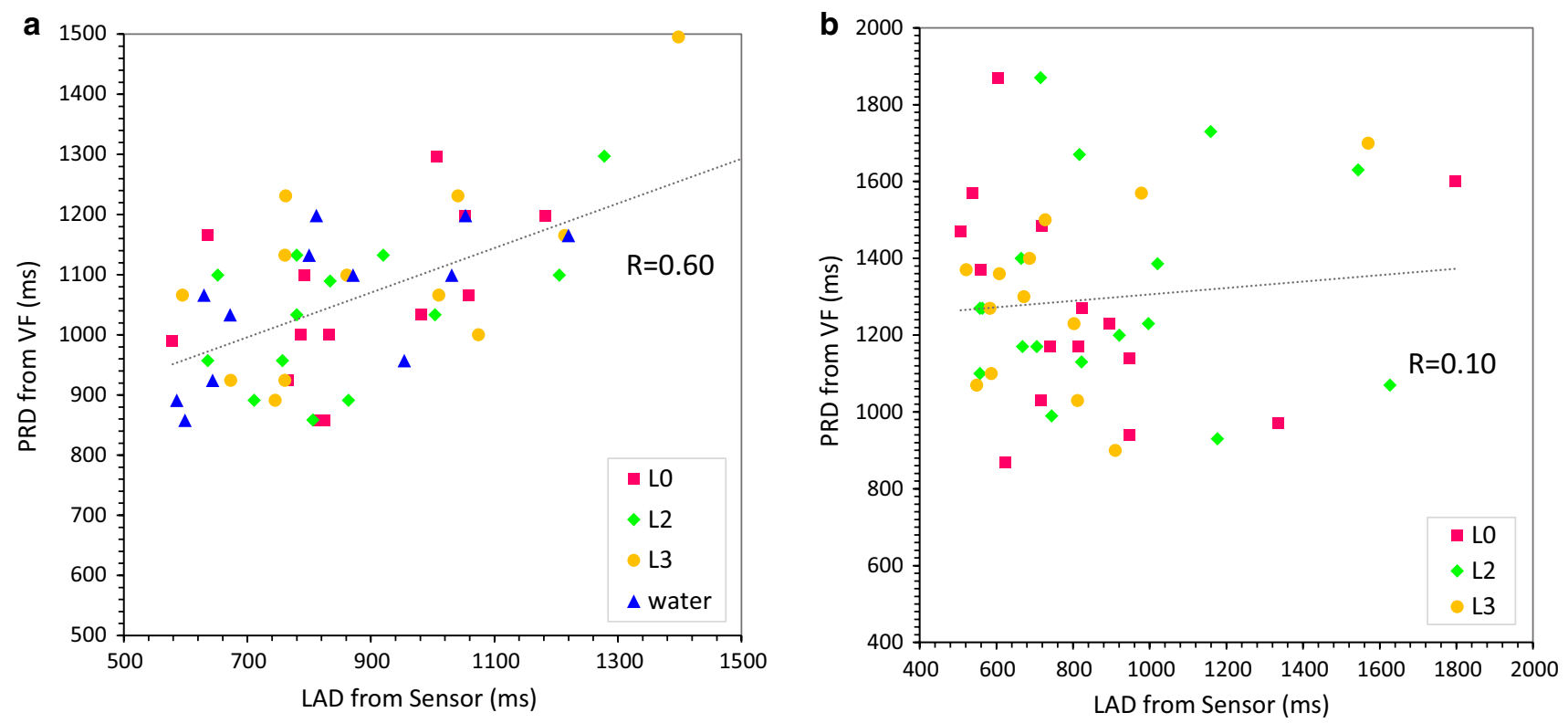

Fig. 9 Comparison between laryngeal activation duration (LAD and pharyngeal response duration (PRD). a Healthy subjects, b patients

healthy subjects and 0.10 in patients, respectively (Fig. 9). Both LEDT and LRT were significantly prolonged in patients with dysphagia $(P<0.0005$ and $P<0.001$, respectively), and the prolongation was more marked in $\mathrm{L} 2$ and L3 test foods.
Figure 10 shows the comparison between ILM of the monitor and the trajectories of the hyoid bone (Fig. 10a) and the thyroid cartilage (Fig. 10b) estimated from VF. Since the hyoid bone is connected to the thyroid cartilage via the thyrohyoid muscle, the hyoid bone dynamics are 


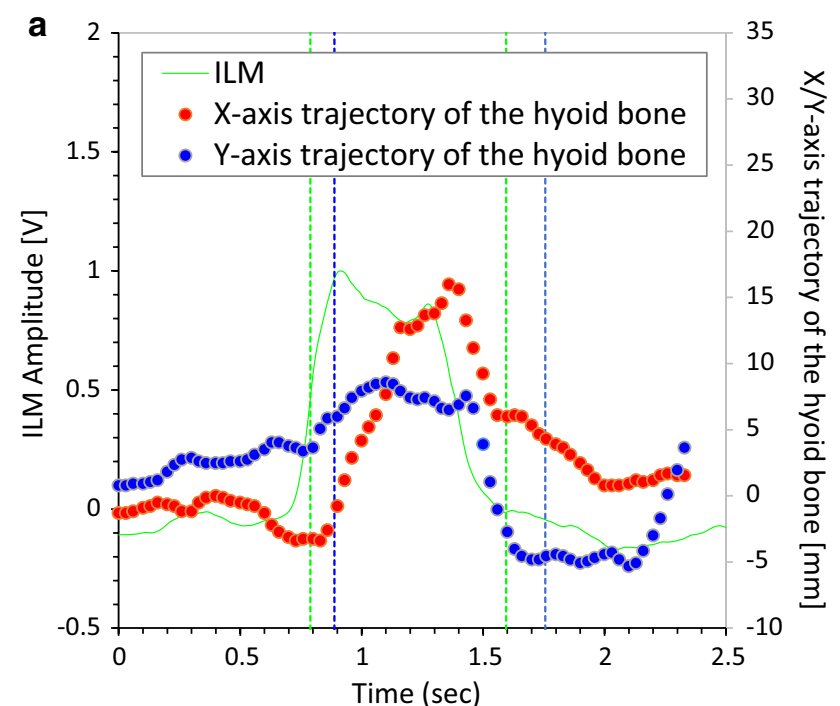

Fig. 10 Comparison of integrated laryngeal motion (ILM) signal and trajectories of the hyoid bone (a) and the vocal cord (b) tracked from videofluoroscopy (VF) images. In each panel, the blue dots are the $X$-axis trajectory, the red dots represent the $Y$-axis trajectory, and the

linked to the thyroid cartilage dynamics. However, they do not completely match, because the activity of the thyrohyoid muscle affects the association of their dynamics. Therefore, we sought to clarify the relationship between PRD and LAD. Simultaneous recordings of VF and the piezoelectric sensor revealed that the onset of hyoid elevation is typically delayed against the positive peak of the piezoelectric signal by $100-200 \mathrm{~ms}$.

\subsection{Temporal relationships between swallowing sound, motion, and respiratory flow}

We analyzed the temporal relationships between swallowing sound, motion, and respiratory flow according to the spatiotemporal and multi-component analyses using the data from simultaneous VF and monitoring. As illustrated in Fig. 5b, the laryngeal ascent often started before the deglutition apnea. The laryngeal motion speed became maximal at $59 \pm 170 \mathrm{~ms}$ (range -229 to $539 \mathrm{~ms}$ ) in healthy subjects and $121 \pm 201 \mathrm{~ms}$ (range -202 to $579 \mathrm{~ms}$ ) in patients from the onset of the deglutition apnea, and Sound I occurred between the onset of laryngeal ascension $\left(T_{1}\right)$ and the instance when the laryngeal motion speed became maximal $(P)$, indicating that Sound I occurs before the pharyngeal swallow. Sound II occurred at $235 \pm 169 \mathrm{~ms}$ (range -125 to $799 \mathrm{~ms}$ ) in healthy subjects and $242 \pm 274 \mathrm{~ms}$ (range -178 to $1513 \mathrm{~ms}$ ) in patients from $P$. Assuming that the pharyngeal swallow starts at the time point $P$, this timing suggests that Sound II occurs at the early stage of the pharyngeal swallow. The tail of the bolus was just below

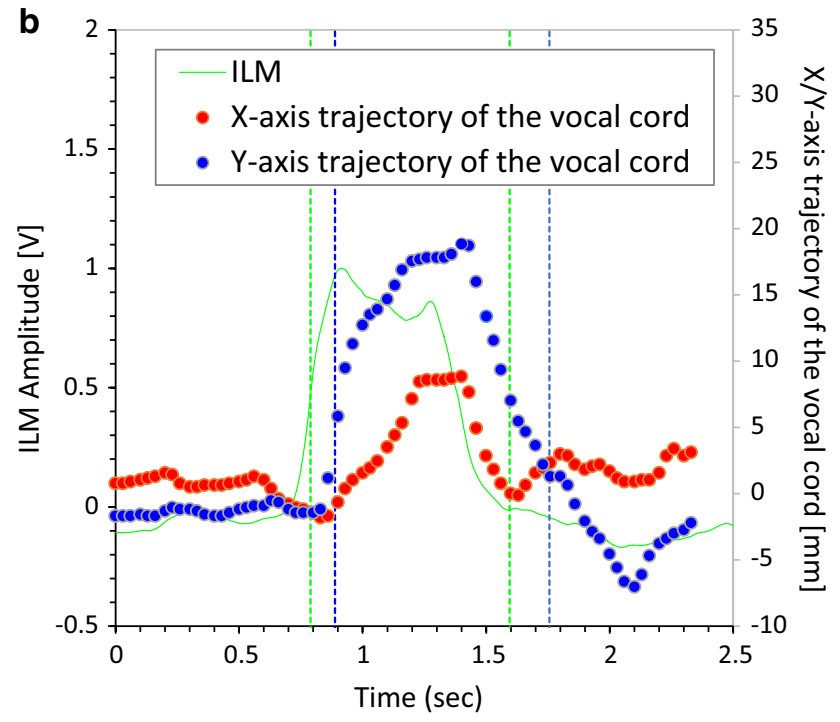

green line is ILM by our monitor device. Start point and end points of laryngeal activation duration (LAD) and pharyngeal response duration (PRD) are shown in green and blue vertical lines, respectively

the upper esophageal sphincter (UES) at the timing when Sound III was heard. When SNIF was observed, Sound III was typically recorded coincident with SNIF, suggesting that Sound III is associated with the relaxation of the pharyngeal constrictor muscle and the closure of UES. ILM reached the trough $T_{2}$ during the descent of the larynx at $25 \pm 456 \mathrm{~ms}$ (range $-1,097$ to $796 \mathrm{~ms}$ ) in healthy subjects and $-348 \pm 584 \mathrm{~ms}$ (range -1359 to $786 \mathrm{~ms}$ ) in patients relative to the end of the deglutition apnea. In healthy subjects, the trough occurred after the end of the deglutition apnea in 63/98 swallows, indicating that respiration was reinitiated during the descent of the larynx in about $64 \%$ of swallows (Fig. 11a). This phenomenon was less marked $(P<0.001)$ in patients.

\section{Discussion}

In the present study, we developed a new swallowing monitoring system that uses respiratory flow, swallowing sound, and laryngeal motion. We found that LAD was moderately correlated with the VF-derived parameter, PRD; however, this correlation was not observed in patients with dysphagia, suggesting that the motion of the hyoid bone and that of the thyroid cartilage were uncoordinated in these patients. On the other hand, although LRT was not correlated with the comparable VF-derived parameter LEDT, LRT was significantly prolonged in patients with dysphagia. Therefore, LRT may also be a useful parameter for detecting dysphagia. Furthermore, the frequency of the I-SW pattern was significantly 

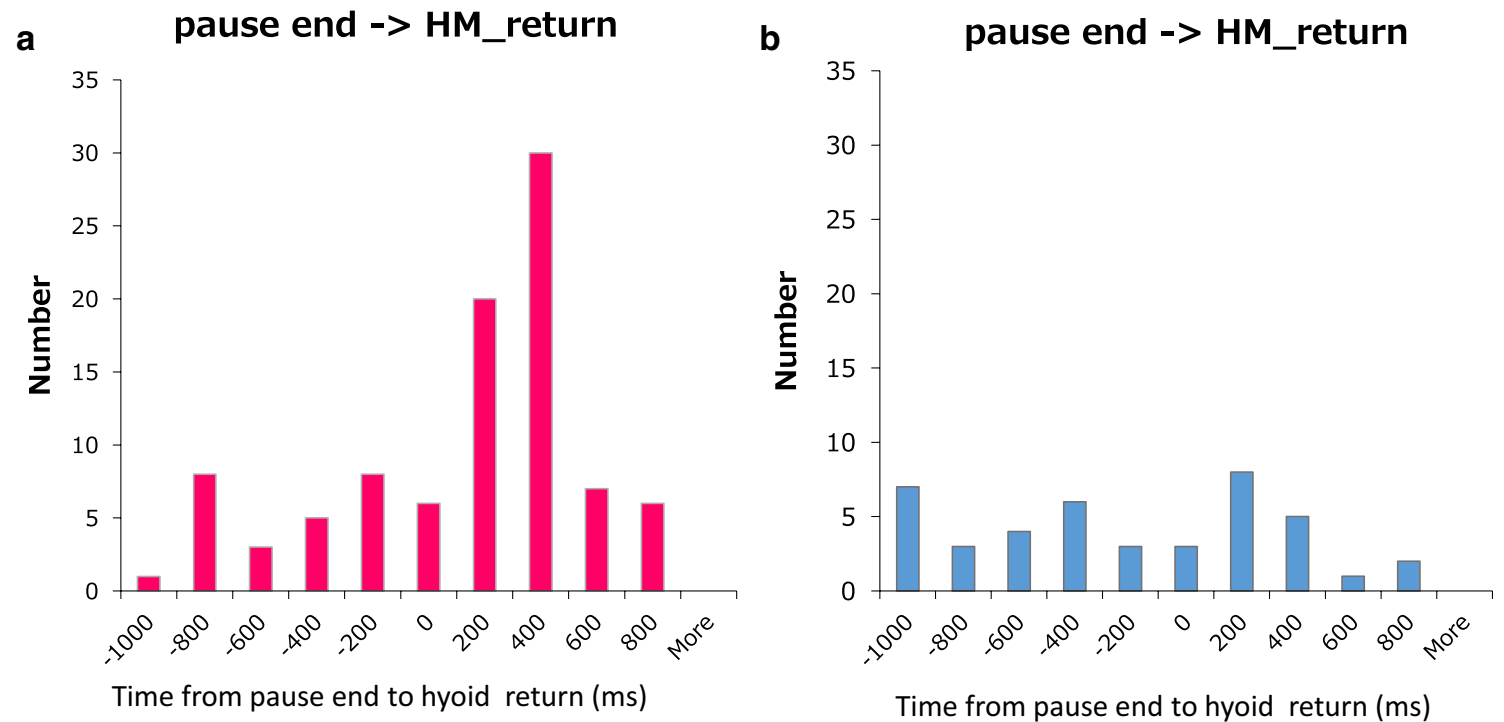

Fig. 11 Timing histogram showing when integrated laryngeal motion (ILM) (the laryngeal position) returns to the trough relative to the deglutition apnea. a Healthy subjects, $\mathbf{b}$ patients

increased in patients with dysphagia. These results suggest that the new device may facilitate the assessment of some aspects of swallowing dysfunction, as well as the detection of aspiration risk in specific patient populations.

\subsection{Consideration of sound component}

The origin of swallowing sound components remains controversial. Vice et al. [46] described the three components of swallowing sound as follows: (1) the initial discrete sound which corresponds to a period when the cricopharyngeus muscle opens, (2) the bolus transit sound which corresponds to the passage of the meal lump to the esophagus, and (3) the final discrete sound which does not always occur. On the other hand, Sato et al. [38] proposed that swallowing sound consists of three sound phases: (1) Sound phase I, may be considered as a closure sound of the epiglottis, (2) Sound phase II, a passage sound through UES, and (3) Sound phase III, an opening sound of the epiglottis. More recently, Moriniere et al. [25] identified three sound components according to the position of the bolus and the anatomic structure in movement: (1) the laryngeal ascension sound when the bolus is located in the oropharynx and/or hypopharynx, (2) the upper esophageal sphincter opening sound where the bolus goes through the sphincter, and (3) the laryngeal release sound when the bolus is located in the esophagus.

In the present study, we also carefully analyzed the timing of sound occurrence and the position of the bolus. We propose a different interpretation regarding the origin of swallowing sound components, as illustrated in Fig. 12: (1) Sound I occurs during the early (preparatory) phase of the laryngeal ascent before the pharyngeal swallow begins. Therefore, we infer

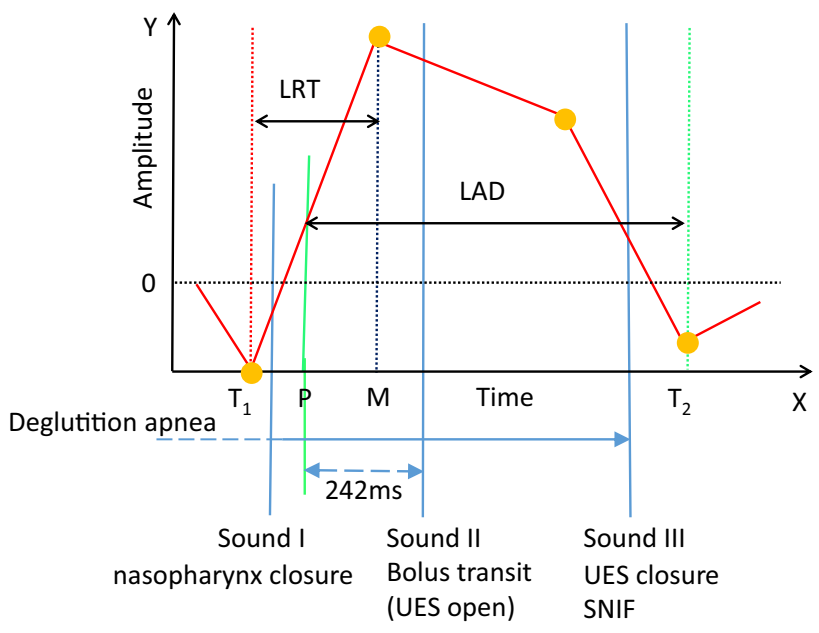

Fig. 12 Positions of swallowing sound components and our speculation of their origins

that Sound I corresponds to the closure of the nasopharynx. The bolus is usually located in the oral cavity, but it can be in the oropharynx or the hypopharynx in the case of the chewswallow complex behavior [21]. (2) Sound II usually occurs 200-250 ms after the onset of the pharyngeal swallow. Since the opening of UES occurs during Sound II, it may contribute to Sound II. However, we think that Sound II is the bolus transit sound mainly through the oropharynx, and the bolus transit sound through UES may not be clearly audible depending on the food consistency, because the tail of the bolus is located just beneath UES at the timing of Sound III, and there is a gap between Sound II and Sound III. In any event, the delay of Sound II from the onset of the pharyngeal swallow indicates 
dysfunction of the pharyngeal phase of swallowing. (3) We agree with the other theories in that Sound III is the laryngeal release, the opening of the nasopharynx, and UES closure sound, because SNIF is observed coincident with Sound III (Fig. 2b, arrowhead). Interestingly, a small negative pressure is also observed coincident with Sound I (Fig. 2b, arrowhead), suggesting that both Sound I and Sound III are sounds associated with the opening or closure of the upper airway.

\subsection{Correspondence between LAD and PRD}

Videofluoroscopic studies have shown that coordinated neuromuscular activity of the mouth, pharynx, larynx and esophagus occurs during swallowing. During swallowing, the larynx is elevated by the contraction of suprahyoid muscles and the thyrohyoid muscle, and the epiglottis covers the laryngeal orifice for airway protection. Although these coordinated activities are generated by a reflex and thus stereotypic, the onset of the pharyngeal swallow is variable in its time of occurrence relative to the position of the bolus [18]. This might be one of the reasons why in the present study LRT was poorly correlated with LEDT (Fig. 9a). In contrast, PRD, which does not depend on the position of the bolus, was moderately correlated with LAD (Fig. 9b). PRD is a temporal parameter associated with the motion of the hyoid bone and estimated from VF images, whereas LAD is a temporal parameter associated with the laryngeal motion, and it is estimated from the piezoelectric sensor signal. Although these two parameters are measured by different systems, they are defined to indicate the same temporal property, i.e., the duration between the onset and the offset of the pharyngeal swallow. The duration of the pharyngeal swallow can be one of the parameters defining the swallowing function, since for example the duration of the pharyngeal swallow is prolonged in COPD patients [4].

In the present study, we evaluated whether the onset and the offset of the swallowing reflex, as measured by the two systems, match by simultaneous recordings. The onset of PRD is the time point when the hyoid bone starts the rapid movement anteriorly and upwardly. This time point was coincident with, or slightly $(-200 \mathrm{~ms})$ delayed relative to the onset of LAD, which is defined as the time point when the upward laryngeal motion reaches the highest speed (Fig. 10). Since the hyoid bone moves by being tracked by the contraction of suprahyoid muscles, there may be a lag between the hyoid bone movement and the muscle contraction. Therefore, the piezoelectric sensor may detect the muscle contraction associated with the laryngeal elevation and consequently respond slightly earlier than the upward hyoid bone movement. Further, the slow frame rate (30 frames/s) may cause an additional time lag.

We defined the offset of PRD as the time point when the larynx returns to the resting position. The reason why we did not choose the time point when the hyoid bone returns to the onset position was that, since the hyoid bone slightly moves upwardly and posteriorly before the onset of the swallowing reflex, the hyoid bone does not return to the onset position. Further, since the resting position of the larynx is determined by the balance between suprahyoid and infrahyoid muscle tones, and these muscle tones are modified by swallowing activity, it was sometimes difficult to judge whether the larynx had returned to the resting position. Indeed, it has been pointed out that the reliability of parameters tracked on videofluorographic images is poor [45]. Therefore, we added an additional constraint that the thyroid cartilage should be at the locally lowest position when the larynx is at the resting position. As a result, the offset of PRD was coincident with, or slightly $(-200 \mathrm{~ms})$ delayed relative to the offset of LAD, which is defined as the time point when the integrated laryngeal motion signal becomes a local minima (Figs. 5, 10). Considering that the inter-rater reliability of temporal VF parameter values, assessed by Cohen's kappa coefficient, ranged between 0.35 and 0.46 [45], we think that the value of the correlation between PRD and LAD in healthy subjects $(r=0.6)$ was reasonable. However, this correlation was disrupted in patients, suggesting that the linkage between the motion of the hyoid bone and that of the larynx is altered in dysphagic patients.

The speed for food to enter into the pharynx depends on the texture. For instance, L2 and L3 foods enter into the pharynx faster than L0 food, and thus, for dysphagic patients, L2 and L3 foods are more difficult to eat than L0 food. Swallowing characteristics change depending on the food texture, and the alteration is more marked in patients [16]. In the present study, we observed that LRT values of patients were prolonged as compared to those of healthy subjects, and this prolongation was more marked in L2 and L3 foods. Therefore, the use of L2 or L3 foods may be preferable to distinguish swallowing abnormality.

\subsection{Coordination between swallowing and breathing}

Swallowing and breathing share a common anatomical pathway in the pharynx. Therefore, the airway must be protected against aspiration by a sequence of laryngeal closure, and a precise coordination between breathing and swallowing was controlled by neuronal networks in the medulla. This coordination is critical during swallowing, and its failure can lead to serious consequences. A normal swallowing activity most frequently occurs during the expiratory phase of the breathing cycle, which interrupts the exhalation movement and the breathing resumes with expiration after swallowing has been completed [40]. However, in elderly persons the chance of swallowing occurrence following inspiration and the chance of post-deglutitive resumption of the respiration being inspiration (not expiration) increase [17, 40]. A similar pattern 
of alteration of the coordination between swallowing and breathing occurs in patients with COPD [10] and Parkinson's disease [9], which may increase the risk of aspiration.

Martin et al. [20] reported that laryngeal elevation follows the onset of respiratory cessation by $0.19 \pm 0.15 \mathrm{~s}$ for water swallows. We also observed that the onset of rapid laryngeal elevation associated with swallowing reflex usually follows the onset of respiratory cessation; however, we found that a preparatory slow laryngeal elevation, during which the closure of the oropharynx occurs, is initiated before deglutition apnea (Fig. 5b). Furthermore, we observed that LRT was greater in dysphagic patients, suggesting that this preparatory slow laryngeal elevation is marked in patients. As to the relationship between laryngeal descent and the termination of respiratory cessation, Martin et al. [20] reported that expiration resumes $0.47 \pm 0.44 \mathrm{~s}$ before the completion of laryngeal descent. We also observed that expiration resumed before the completion of laryngeal descent in a majority of the healthy subjects (Fig. 11a). However, such a phenomenon was less evident in the patients (Fig. 11b). The physiological significance of expiration before the completion of laryngeal descent remains unclear and necessitates further exploration in the future.

The coordination between swallowing and breathing occurs by the interaction of central pattern generators (CPGs) for swallowing and breathing within the brainstem [7, 30]. Bautista et al. [2] proposed that balanced synaptic interaction along the nucleus of the solitary tract (NTS)/ Kölliker-Fuse (KF) nucleus axis is pivotal for effective swallowing/breathing coordination, and an imbalance of the synaptic interaction between and within NTS and KF may have an important role in the pathophysiology of swallowing disorders. In the present study, similar to the cases of COPD [10] and Parkinson's disease [9], the I-SW pattern was more frequently observed in patients with dysphagia. Thus, we suggest that the disordered coordination between swallowing and breathing may be a sensitive and early indicator of a functional abnormality of swallowing CPG and/or an interaction between swallowing and respiratory CPGs. In addition, altered properties of peripheral effector organs, e.g., lung function impairments, would profoundly affect the coordination between swallowing and breathing. Interestingly, CPAP improves respiration-swallowing coordination during sleep [37], and the improvement in respiratory-swallowing coordination results in favorable effects on airway protection and bolus clearance [19]. Therefore, detection of discoordination between breathing and swallowing may lead to early intervention for asymptomatic patients to prevent aspiration.

Swallows can be viewed as external stimuli to the respiratory CPG. The respiratory rhythm is reset by a swallow, and the respiratory phase is shifted. The amount of shift depends on the timing when the swallow occurs within the respiratory cycle. Such phase-response characteristics reflect the internal structure of the respiratory CPG as well as the properties of relay pathways and effector organs (diaphragm, lung, and chest wall) [29, 31]. Paydarfar et al. [32] reported that the interval between the swallowing event and the onset of inspiration is the shortest when swallowing occurs at the E-I transition and largest when swallowing occurs at the I-E transition, in the case of water swallowing. Therefore, swallows at early expiration are the safest with regard to the risk of aspiration. We observed similar phase-response characteristics in both the healthy subjects and patients. The interval between swallowing and subsequent inspirations was highly variable for swallows which occurred near the I-E transition. This may result from the difference in food consistency. In case of level 0 and level 2 test foods (jelly consistency), the interval tended to be prolonged (Fig. 8). Further study is necessary to elucidate factors altering the variation in the phase-response near the I-E transition.

In addition to the autonomic regulation, voluntary and behavioral controls by higher brain centers may affect the coordination between laryngeal motion and breathing activity. For instance, anticipation of the speed of bolus movement may advance or delay the onset of slow laryngeal ascension before the pharyngeal swallow, because depending on the food consistency, subjects can predict the speed of bolus passing through the oropharynx based on their experience. On the other hand, the residue awareness may delay the laryngeal relaxation to prepare for a dry swallowing, or to clear the residual food.

\subsection{Technical considerations}

In the present study, we adopted a semiautomatic swallowing detection method. The reason why we adopted a semiautomatic rather than a full-automatic detection method was that the sensitivity must be almost $100 \%$ for the clinical assessment of swallowing function. It was reported that the accuracy of the full-automatic swallowing detection method was 82-85 \% [8, 39], and we also achieved a similar accuracy; however, it was not sufficient. Although the present study was done while the participants were awake, the device may be used to monitor swallowing while subjects are asleep. Therefore, in a practical situation, several artifacts such as head movement, talking, snoring and electrical interference may further deteriorate the accuracy of swallowing activity detection. In addition, mouth breathing, often observed during the chew-swallow complex behavior, may blunt the respiratory flow signal captured by the nasal cannula-type flow sensor, thereby obscuring the expiratory flow. Therefore, we adopted the semiautomatic detection method to pick up all swallows. We found that the inter-rater variability judged from played back sound and displayed laryngeal motion was extremely small (kappa coefficient $=1.0$ ). 
We set the cutoff frequency at $100 \mathrm{~Hz}$ to divide sound and motion components. This cutoff frequency might be too high, because the low-frequency component contains both laryngeal motion signal and low-frequency $(20-100 \mathrm{~Hz})$ acoustic signal. Lee et al. [14] reported that most of the signal energy measured by accelerometry is contained in the low frequencies, approximately below $100 \mathrm{~Hz}$. However, they suggested that the accelerometry signal may be primarily attributed to a mechanical rather than acoustic phenomenon. On the other hand, swallowing sound typically contains a high-frequency component of greater than $750 \mathrm{~Hz}$ (see Fig. 1 in Sazonov et al. [39]), and Sarraf-Shirazi et al. [35] use the $100 \mathrm{~Hz}$ cutoff frequency to characterize the swallowing sounds recorded in the ear, nose, and on trachea. Therefore, we assumed that this high $(>100 \mathrm{~Hz})$ frequency component is essential in discriminating the swallowing sound from environmental noises. Indeed, speech therapists were able to accurately discriminate swallows by playing back piezoelectric signals above $100 \mathrm{~Hz}$. Therefore, we think that the signal above $100 \mathrm{~Hz}$ captures important features of the swallowing sound; however, the cutoff frequency can be optimized by future development.

\subsection{Study limitation}

Obviously, the sample size in the present study is insufficient to draw a promising conclusion. Further data collection from healthy subjects as well as patients with dysphagia is necessary to improve the detection algorithm and to define normal swallows. In addition, a full-automatic detection method, such as one using pattern recognition methods, needs to be developed in the future.

The coordination between breathing and swallowing is important in the detection of aspiration, although this study was not designed to investigate the effect of aspiration on the parameters. Further study is necessary to elucidate whether the discoordination between breathing and swallowing detects an aspiration event and/or predicts the risk of aspiration.

\section{Conclusions}

In this study, we proposed a novel sound, motion, and air flow recognition technique to detect swallowing events and assess the swallowing function. To our knowledge, this is the first bedside swallowing monitoring system that can assess the swallowing sound, the laryngeal motion during swallowing, and the coordination between swallowing and breathing in a systematic manner. With the device developed in the present study, swallowing activity is semiautomatically detected at a high sensitivity, and the quality of swallows can be assessed from various aspects, i.e., the duration of swallowing reflex, the timing of swallowing sound relative to the laryngeal motion, and the coordination between breathing and swallowing. Therefore, the new device may facilitate the assessment of some aspects of swallowing dysfunction, especially with respect to the coordination between swallowing and breathing.

Acknowledgments We thank Prof. Akira Ishikawa of Kobe University and Dr. Hajime Takahashi, the President of Takahashi Hospital for providing opportunity to acquire videofluoroscopic data, Prof. Jun Kayashita and Dr. Yoshie Yamagata of Hiroshima Prefectural University for providing test foods, Hiroshi Ueno and Hiroyuki Takeda of J Craft Co., Ltd. for constructing the 'swallowing simulator,' Dr. Masako Kijima of Wakakusa rehabilitation hospital, Kayoko Ohtsuka, SLP of Yamato University and Yuri Katsuta, SLP of Wakakusa rehabilitation hospital for helping to obtain patient data, and Prof. Michiaki Mishima and Prof. Ryosuke Takahashi of Kyoto University for giving us critical comments. This work was supported by Grantin-Aid for Scientific Research(C) 16K01546.

\section{Compliance with ethical standards}

Conflict of interest This study has been conducted with funding support of Foodcare Co., Ltd. and J Craft Co., Ltd.

Open Access This article is distributed under the terms of the Creative Commons Attribution 4.0 International License (http://creativecommons.org/licenses/by/4.0/), which permits unrestricted use, distribution, and reproduction in any medium, provided you give appropriate credit to the original author(s) and the source, provide a link to the Creative Commons license, and indicate if changes were made.

\section{References}

1. Aboofazeli M, Moussavi Z (2004) Automated classification of swallowing and breadth sounds. Conf Proc IEEE Eng Med Biol Soc 5:3816-3819. doi:10.1109/IEMBS.2004.1404069

2. Bautista TG, Dutschmann M (2014) Ponto-medullary nuclei involved in the generation of sequential pharyngeal swallowing and concomitant protective laryngeal adduction in situ. J Physiol 592:2605-2623. doi:10.1113/jphysiol.2014.272468

3. Brodsky MB, McFarland DH, Michel Y, Orr SB, MartinHarris B (2012) Significance of nonrespiratory airflow during swallowing. Dysphagia 27:178-184. doi:10.1007/ s00455-011-9350-4

4. Cassiani RA, Santos CM, Baddini-Martinez J, Dantas RO (2015) Oral and pharyngeal bolus transit in patients with chronic obstructive pulmonary disease. Int J Chronic Obstr 10:489-496. doi:10.2147/Copd.S74945

5. Crary MA, Sura L, Carnaby G (2013) Validation and demonstration of an isolated acoustic recording technique to estimate spontaneous swallow frequency. Dysphagia 28:86-94. doi:10.1007/ s00455-012-9416-y

6. Davis S, Mermelstein P (1990) Comparison of parametric representations for monosyllabic word recognition in continuously spoken sentences. Acoust Speech Signal Process IEEE Trans 28:357-366. doi:10.1109/TASSP.1980.1163420

7. Dick TE, Oku Y, Romaniuk JR, Cherniack NS (1993) Interaction between central pattern generators for breathing and swallowing in the cat. J Physiol 465:715-730 
8. Golabbakhsh M, Rajaei A, Derakhshan M, Sadri S, Taheri M, Adibi P (2014) Automated acoustic analysis in detection of spontaneous swallows in Parkinson's disease. Dysphagia 29:572-577. doi:10.1007/s00455-014-9547-4

9. Gross RD, Atwood CW Jr, Ross SB, Eichhorn KA, Olszewski JW, Doyle PJ (2008) The coordination of breathing and swallowing in Parkinson's disease. Dysphagia 23:136-145

10. Gross RD, Atwood CW Jr, Ross SB, Olszewski JW, Eichhorn KA (2009) The coordination of breathing and swallowing in chronic obstructive pulmonary disease. Am J Respir Crit Care Med 179:559-565

11. Kim Y, McCullough GH (2008) Maximum hyoid displacement in normal swallowing. Dysphagia 23:274-279. doi:10.1007/ s00455-007-9135-y

12. Kunieda K, Ohno T, Fujishima I, Hojo K, Morita T (2013) Reliability and validity of a tool to measure the severity of dysphagia: the Food Intake LEVEL Scale. J Pain Symptom Manage 46:201-206. doi:10.1016/j.jpainsymman.2012.07.020

13. Langmore SE, Skarupski KA, Park PS, Fries BE (2002) Predictors of aspiration pneumonia in nursing home residents. Dysphagia 17:298-307. doi:10.1007/s00455-002-0072-5

14. Lee J, Steele CM, Chau T (2008) Time and time-frequency characterization of dual-axis swallowing accelerometry signals. Physiol Meas 29:1105-1120. doi:10.1088/0967-3334/29/9/008

15. Lof GL, Robbins J (1990) Test-retest variability in normal swallowing. Dysphagia 4:236-242

16. Logemann JA, Shanahan T, Rademaker AW, Kahrilas PJ, Lazar R, Halper A (1993) Oropharyngeal swallowing after stroke in the left basal ganglion/internal capsule. Dysphagia 8:230-234

17. Martin-Harris B, Brodsky MB, Michel Y, Ford CL, Walters B, Heffner J (2005) Breathing and swallowing dynamics across the adult lifespan. Arch Otolaryngol Head Neck Surg 131:762-770. doi:10.1001/archotol.131.9.762

18. Martin-Harris B, Brodsky MB, Michel Y, Lee FS, Walters B (2007) Delayed initiation of the pharyngeal swallow: normal variability in adult swallows. J Speech Lang Hear Res 50:585-594. doi:10.1044/1092-4388(2007/041)

19. Martin-Harris B, McFarland D, Hill EG, Strange CB, Focht KL, Wan Z, Blair J, McGrattan K (2015) Respiratory-swallow training in patients with head and neck cancer. Arch Phys Med Rehabil 96:885-893. doi:10.1016/j.apmr.2014.11.022

20. Martin BJ, Logemann JA, Shaker R (1985) Dodds WJ (1994) Coordination between respiration and swallowing: respiratory phase relationships and temporal integration. J Appl Physiol 76:714-723

21. Matsuo K, Palmer JB (2015) Coordination of oro-pharyngeal food transport during chewing and respiratory phase. Physiol Behav 142:52-56. doi:10.1016/j.physbeh.2015.01.035

22. Merey C, Kushki A, Sejdic E, Berall G, Chau T (2012) Quantitative classification of pediatric swallowing through accelerometry. J Neuroeng Rehabil 9:34. doi:10.1186/1743-0003-9-34

23. Miyaji H, Umezaki T, Adachi K, Sawatsubashi M, Kiyohara H, Inoguchi T, To S, Komune S (2012) Videofluoroscopic assessment of pharyngeal stage delay reflects pathophysiology after brain infarction. Laryngoscope 122:2793-2799. doi:10.1002/lary.23588

24. Mokhlesi B, Logemann JA, Rademaker AW, Stangl CA, Corbridge TC (2002) Oropharyngeal deglutition in stable COPD. Chest 121:361-369

25. Moriniere S, Boiron M, Alison D, Makris P, Beutter P (2008) Origin of the sound components during pharyngeal swallowing in normal subjects. Dysphagia 23:267-273. doi:10.1007/s00455-007-9134-z

26. Nagasaka Y (2012) Lung sounds in bronchial asthma. Allergol Int 61:353-363. doi:10.2332/allergolint.12-RAI-0449

27. Nikjoo MS, Steele CM, Sejdic E, Chau T (2011) Automatic discrimination between safe and unsafe swallowing using a reputation-based classifier. Biomed Eng Online 10:100. doi:10.1186/1475-925X-10-100
28. Nishino T (2012) The swallowing reflex and its significance as an airway defensive reflex. Front Physiol 3:489. doi:10.3389/ fphys.2012.00489

29. Oku Y, Dick TE (1992) Phase resetting of the respiratory cycle before and after unilateral pontine lesion in cat. J Appl Physiol 72:721-730

30. Oku Y, Tanaka I, Ezure K (1994) Activity of bulbar respiratory neurons during fictive coughing and swallowing in the decerebrate cat. J Physiol 480:309-324

31. Paydarfar D, Eldridge FL, Kiley JP (1986) Resetting of mammalian respiratory rhythm: existence of a phase singularity. Am J Physiol 250:R721-R727

32. Paydarfar D, Gilbert RJ, Poppel CS, Nassab PF (1995) Respiratory phase resetting and airflow changes induced by swallowing in humans. J Physiol 483(Pt 1):273-288

33. Robinson DJ, Jerrard-Dunne P, Greene Z, Lawson S, Lane S, O'Neill D (2011) Oropharyngeal dysphagia in exacerbations of chronic obstructive pulmonary disease. Eur Geriatr Med 2:201203. doi:10.1016/j.eurger.2011.01.003

34. Santamato A, Panza F, Solfrizzi V, Russo A, Frisardi V, Megna M, Ranieri M, Fiore P (2009) Acoustic analysis of swallowing sounds: a new technique for assessing dysphagia. J Rehabil Med 41:639-645. doi:10.2340/16501977-0384

35. Sarraf-Shirazi S, Baril JF, Moussavi Z (2012) Characteristics of the swallowing sounds recorded in the ear, nose and on trachea. Med Biol Eng Comput 50:885-890. doi:10.1007/s11517-012-0938-0

36. Sarraf Shirazi S, Buchel C, Daun R, Lenton L, Moussavi Z (2012) Detection of swallows with silent aspiration using swallowing and breath sound analysis. Med Biol Eng Comput 50:1261-1268. doi:10.1007/s11517-012-0958-9

37. Sato K, Umeno H, Chitose S, Nakashima T (2011) Sleep-related deglutition in patients with OSAHS under CPAP therapy. Acta Otolaryngol 131:181-189. doi:10.3109/00016489.2010.520166

38. Sato T, Komai K, Kato K, Kawashima N, Agishi T, Akamatsu M, Ando T (2005) Diagnosis of swallowing difficulties using time-frequency analysis of swallowing sound signals based on wavelet transformation. ASAIO J 51:15A. doi:10.1097/00002480-200503000-00058

39. Sazonov ES, Makeyev O, Schuckers S, Lopez-Meyer P, Melanson EL, Neuman MR (2010) Automatic detection of swallowing events by acoustical means for applications of monitoring of ingestive behavior. IEEE Trans Bio Med Eng 57:626-633

40. Shaker R, Li Q, Ren J, Townsend WF, Dodds WJ, Martin BJ, Kern MK, Rynders A (1992) Coordination of deglutition and phases of respiration: effect of aging, tachypnea, bolus volume, and chronic obstructive pulmonary disease. Am J Physiol 263:G750-G755

41. Shelton RL Jr, Bosma JF, Sheets BV (1960) Tongue, hyoid and larynx displacement in swallow and phonation. J Appl Physiol 15:283-288

42. Shieh WY, Wang CM, Chang CS (2015) Development of a portable non-invasive swallowing and respiration assessment device. Sensors (Basel) 15:12428-12453. doi:10.3390/s150612428

43. Terada K, Muro S, Ohara T, Kudo M, Ogawa E, Hoshino Y, Hirai T, Niimi A, Chin K, Mishima M (2010) Abnormal swallowing reflex and COPD exacerbations. Chest 137:326-332

44. Teramoto S, Sudo E, Matsuse T, Ohga E, Ishii T, Ouchi Y, Fukuchi Y (1999) Impaired swallowing reflex in patients with obstructive sleep apnea syndrome. Chest 116:17-21. doi:10.1378/chest.116.1.17

45. Tohara H, Nakane A, Murata S, Mikushi S, Ouchi Y, Wakasugi Y, Takashima M, Chiba Y, Uematsu H (2010) Inter- and intra-rater reliability in fibroptic endoscopic evaluation of swallowing. J Oral Rehabil 37:884-891. doi:10.1111/j.1365-2842.2010.02116.x

46. Vice FL, Heinz JM, Giuriati G, Hood M, Bosma JF (1990) Cervical auscultation of suckle feeding in newborn infants. Dev Med Child Neurol 32:760-768

47. Yagi N, Takahashi R, Ueno H, Yabe T, Oke Y, Oku Y (2014) Swallow-monitoring system with acoustic analysis for dysphagia. 
In: Paper presented at the IEEE International Conference on Systems, Man and Cybernetics (SMC), San Diego, CA, 5-8 Oct

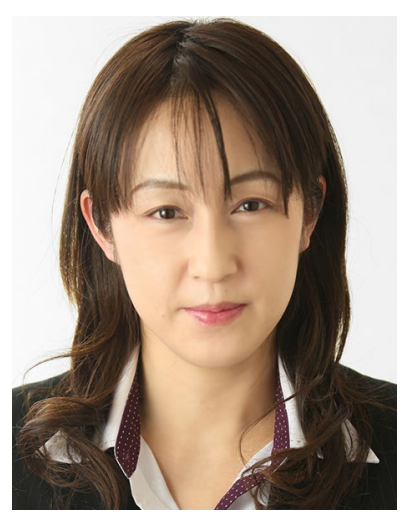

Naomi Yagi is Ph.D. of Engineering and a Program-Specific Researcher in Graduate School of Medicine, Kyoto University. Her interests include medical informatics.

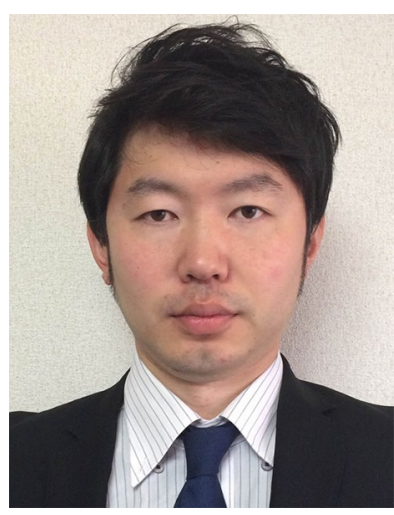

Shinsuke Nagami is Speech therapist and a Swallowing rehabilitation-Specific Researcher, Graduate School of Medicine, Kyoto University.

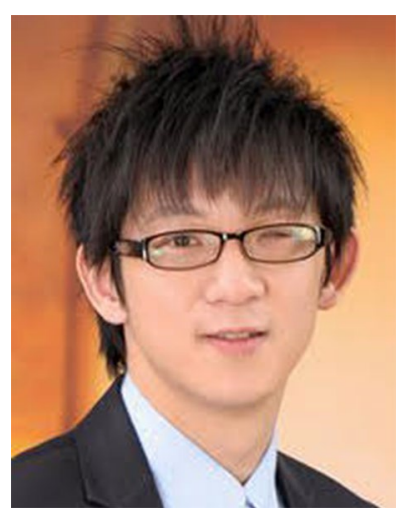

Meng-kuan Lin received Ph.D. in Medical Informatics from USQ, Australia, in 2012. $\mathrm{He}$ is currently working as a scientist in BSI, Riken. His research interests include neuroscience and signal processing.

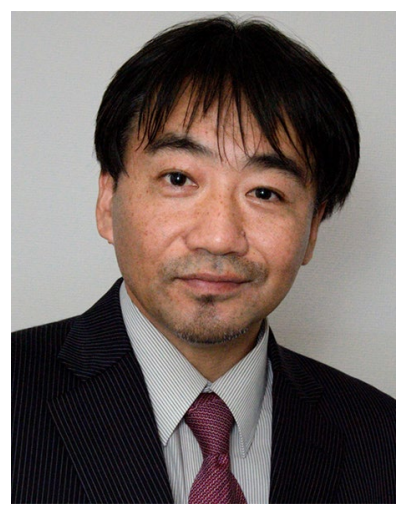

Toru Yabe a mechanical engineer at Murata Manufacturing Co., Ltd., is developing the piezoelectric sensor system.

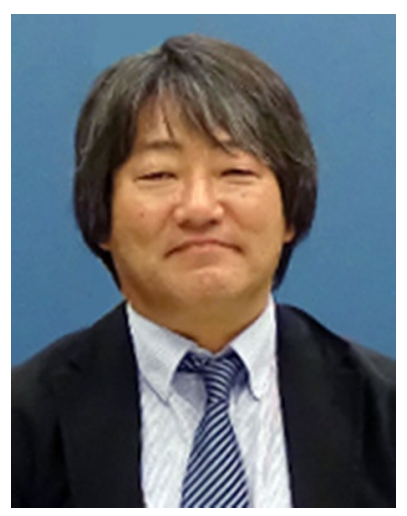

Masataka Itoda is Dentist and received Ph.D. Degree in Osaka Dental University (Fixed Prosthodontics and Occlusion). His research interest includes masticatory disturbance and swallowing disorder.

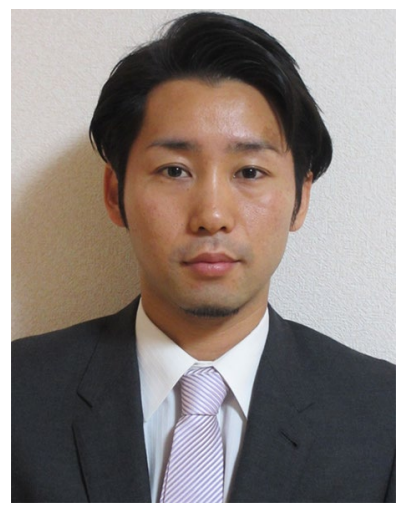

Takahisa Imai is Speech therapist, Department of Rehabilitation, Ashiya Municipal Hospital.

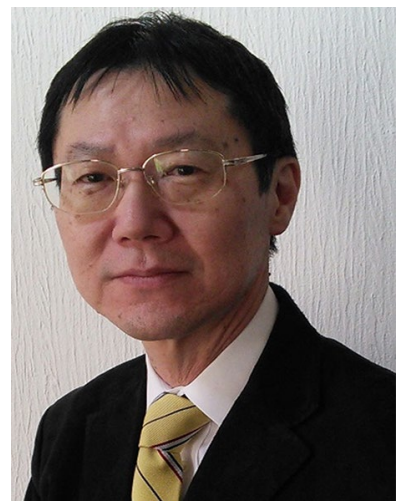

Yoshitaka Oku is Professor of Department of Physiology, Hyogo College of Medicine. He is directing a translational research project 'Swallowing Project'. His research interests are breathing and swallowing physiology. 